\title{
ECN Pressure and \\ Vacuum Vessel Engineering Notes
}

\author{
3740.224 - EN - 323 \\ K. Dixon/J. Wu \\ October 17, 1991
}

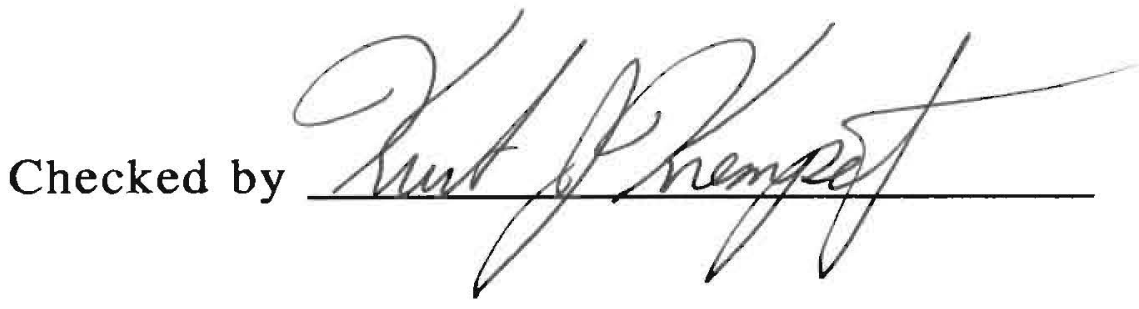


PRESSURE VESSEL ENGINEERING NOTE

PER MANDATORY STANDARD SD37

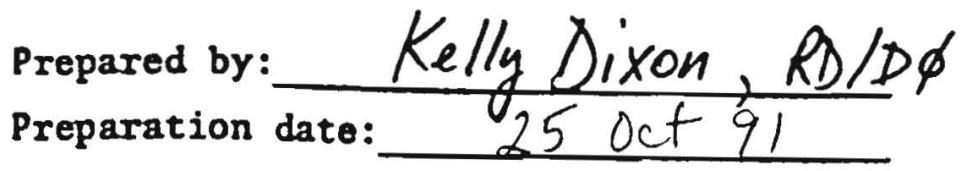

1 Description and Identification

Fill in the label information below:

This vessel conforms to engineering standard SD37

Vesse1 Titio Nurth Endcap Calorimeter (NEC) Cryostat

vessel Number RD 4025

Vesse1 Drawing Number $3740.224-$ ME- 273065

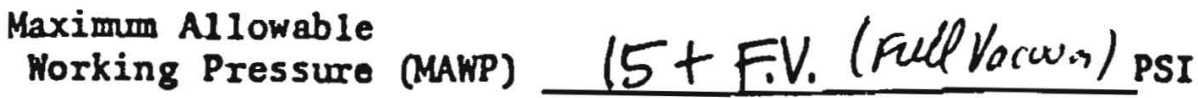

Working Temperature Range $-298{ }_{F}+100{ }_{F}$

Contents Liguid argon, calorimeter modules, instrumentation

Designer/Manufacturer Precess Engineering The.

Test Pressure (if tested at Fermi)

40 PSI, Hydraulic

Acceptance

Dote: 25June 91

Pneumatic

Obtain from

Division/Section

Safety Officer
Actual signature required in this space

of Division/Section Research DNAsen 250 TP/

NOTE: Any subsequent changes in contents, pressures, temperatures, valving, etc., which affect the safety of

this vessel shall require another review and test.

Reviewed by: hy C Y Reslacke

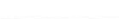

Accepted as fonforming to standard by

conform to the requirements of the standard.

Date:

Lab Property Number(s):

Lab Location Code: $\quad$ DAB (obtain from Safety Officer)

Purpose of Vesse1 (s): Liguid argon calorimeter for D $\phi$ Colliding Detector

Vessel Capacity/Size: $1200 \mathrm{ft}^{3} \mathrm{vo} / 0$ modules oiameter : $1924^{\prime \prime}$ Length: $95^{\prime \prime}$ Normal Operating Pressure (OP) PSI

MAWP-OP = 10 PSI

Is the above enough to provide rolief cracking pressure tolerance plus system uncertainty tolerance per M-9. Yes

As an option, provide a photo of the entire vessel in the Appendix. 
List the numbers of all pertinent drawings and the location of the originals.

(Append copies).

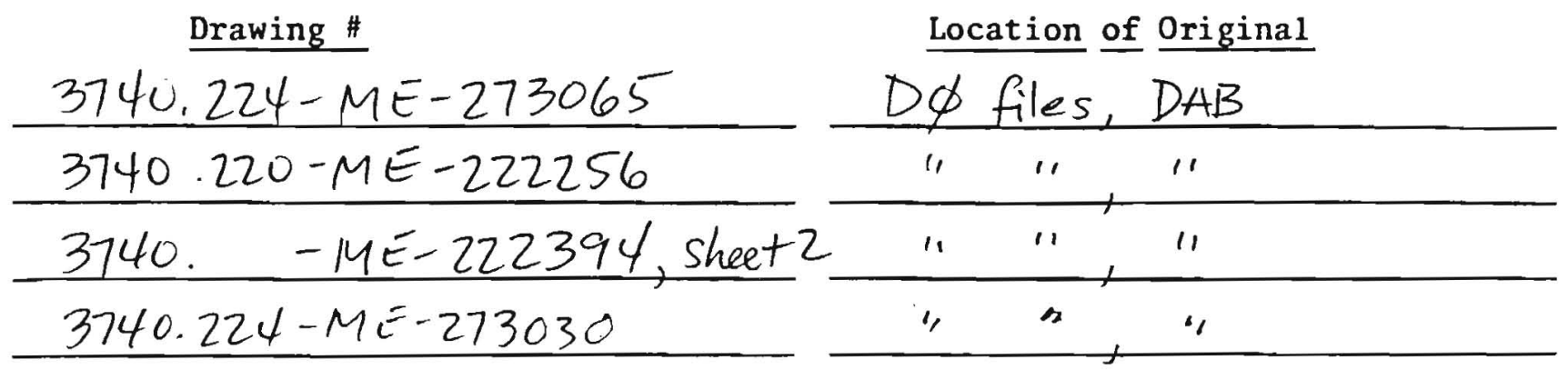

2 Design Verification

Does the vessel(s) have a U stamp? Yes $X$ No___. If "Yes", fill out data below and skip page 3 ; if "No", fill out page 3 and skip this page.

Staple photo of U stamp plate below.

Copy "U" label details to the side if photo

is not clear of if copies are unreadable.

Copy data here:

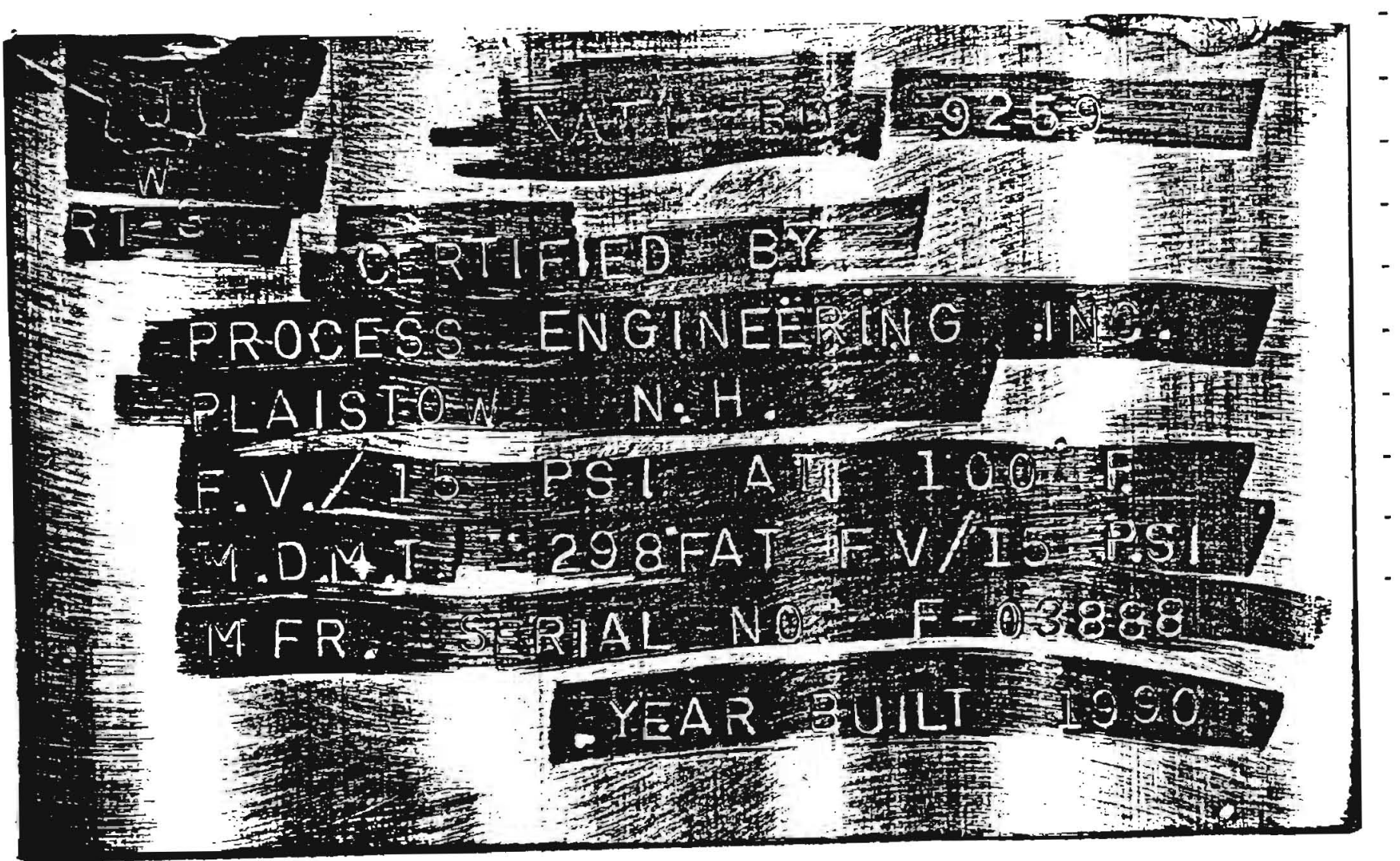


On the sketch below, circle all applicable sections of the ASME code per Section VIII, Division I. List the results of all calculations. (Insert copies of calculations in the appendix).

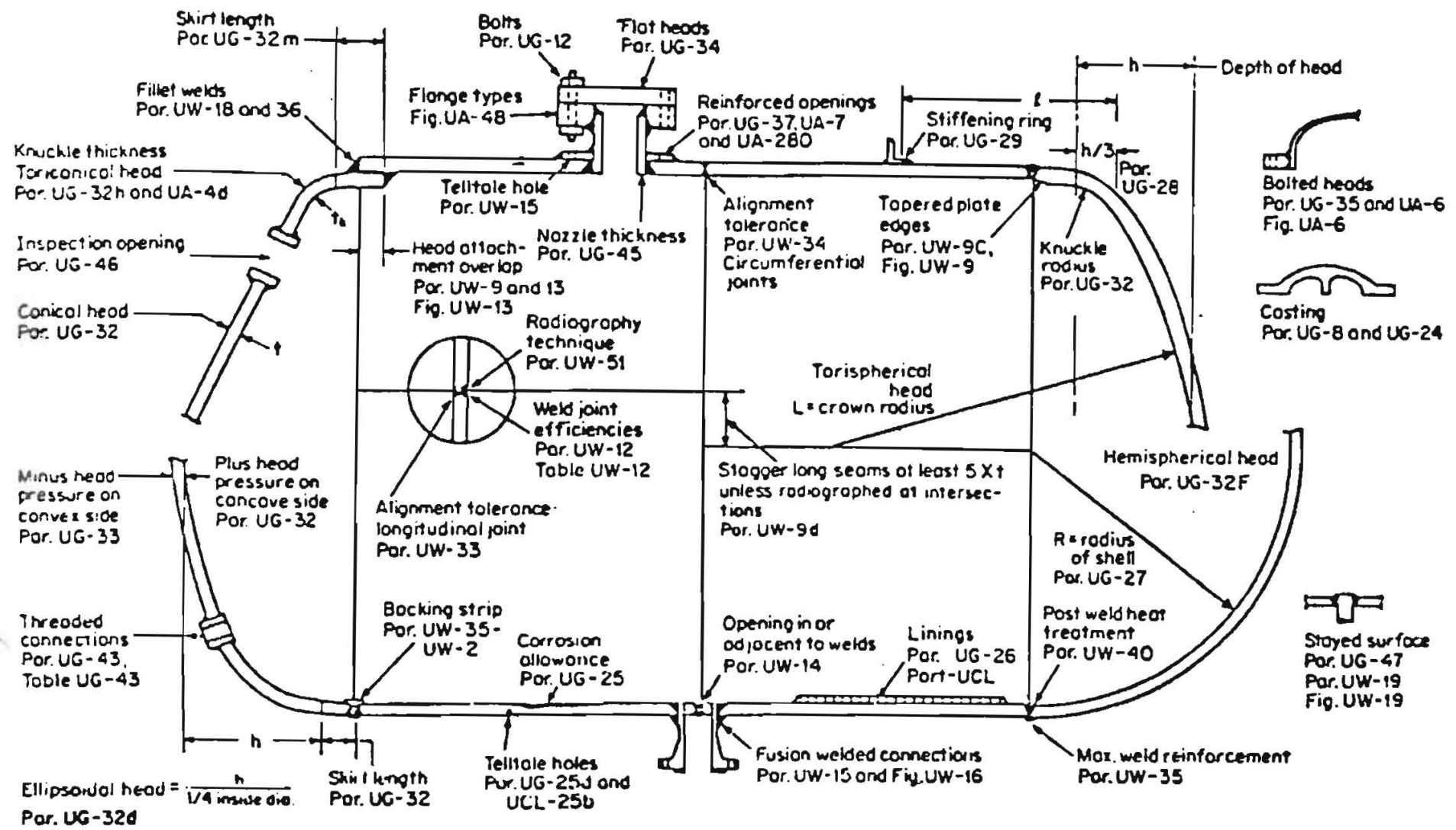

Summary of ASME Code \& Related Design References

Item

Design Summary - EC Cryostats

EC Contract including design spec. \& Battelle review

ASME Code Data Report

Siructural Evaluation of the EC Cryostat Stanchions 3740.224-EN-216
DфEN\#

$3740.224-E N-194$

$3740.224-E N-325$

$3740.224-E N-326$

If this vessel is exceptional or had exceptional parts, list their details under 5.6. Yes No 
3 System Venting. Provide the system schematic in the Appendix, if the vessel safety is system sensitive.

Is it possible to isolate the relief valves by a valve from the vessel?

Yes No $X^{*}$

If "Yes", the system must conform to M-5. Provide an explanation on the appended schematic. (An isolatable vessel, not conforming to $M-5$ violates the Standard.)

Is the relief cracking pressure set at or below the M.A.W.P.?

Yes $X$ No Actual setting 13 PSI

(A no response violates the Standard.)

Is the pressure drop of the relief system at maximum anticipated flow such that vessel pressure never rises above the following? (UG 125)

Yes $X$ No $110 \%$ of MAWP (one relief)

$116 \%$ of MAWP (multiple reliefs)

$121 \%$ of MAWP (unexpected heat source)

Provide test or calculational proof in the Appendix.

(Non-conforming pressure rises violate the Standard.)

List of reliefs and settings:

Manufacturer

AGCO

Fike Metal Products

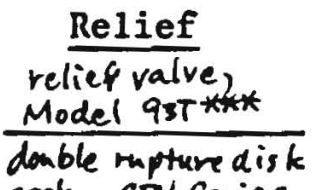

ass'y, CPV Series

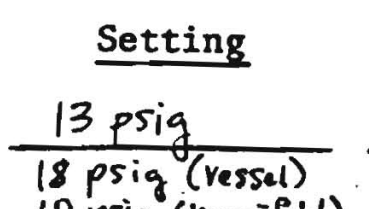

10 prig (manifild)

Is the relief device an ASME stamped device? Yes

No

Flow Rate ${ }^{* *} \quad \underline{\text { Size }}$

760 scfm air $2^{\prime \prime} \times 3^{\prime \prime}$

$850 \mathrm{Scfmair}$
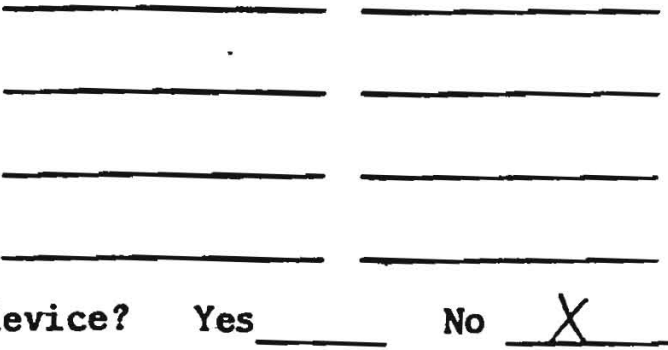

4 Operating Procedure

Is an operating procedure necessary for the safe operation of this vessel?

Yes $X$ No - If "Yes", please append.

Welding Information

Has the vessel been fabricated in a Fermilab shop? Yes No $X$

If "Yes", append a copy of the welding shop statement of welder qualification and a copy of the Welding Procedure Specification (WPS) used to weld this vessel.

Exceptional, Existing, Used, and Non-Mamed Area Vessels

Is this vessel or any part thereof in the above categories? Yes

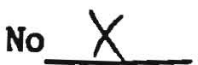

If "Yes", follow the Engineering Note requirements for documentation in free form below.

- Block Valve is on the DISCHARGE side of the relief valve. See EN-155 for further information.

* Evaluated at $96 \mathrm{~K}$ inlet temperature. Flow rates are down rated for system effects (upstream and downstream pressure drops).

... Pilot operated type; sense line from rupture disk inlet. 
Fermilab

EXHIBIT B

Pressure Testing Permit*

Type of Test:

Test Pressure:

Hydrostatic $4 l^{\prime} \quad$ psig

Pneumatic

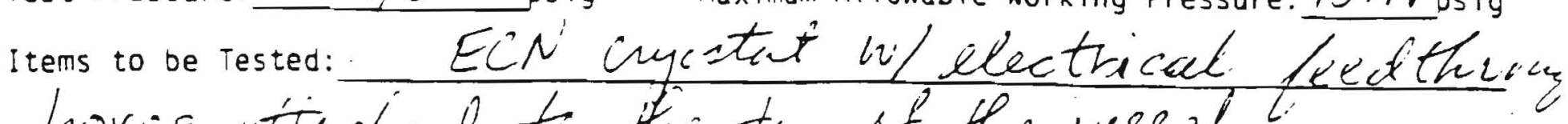

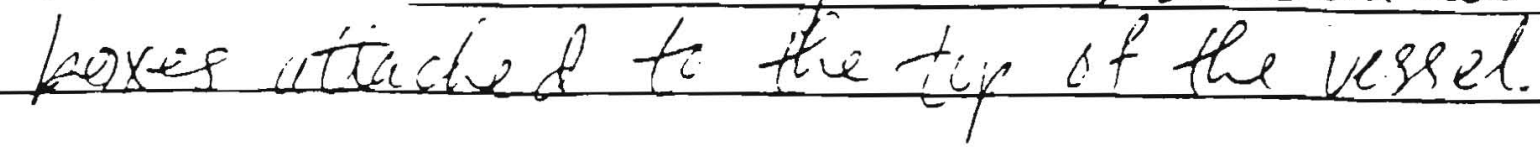

Location of Test: Nirth Sideculik/DAB Date and Time: 7/17/91,5 PM

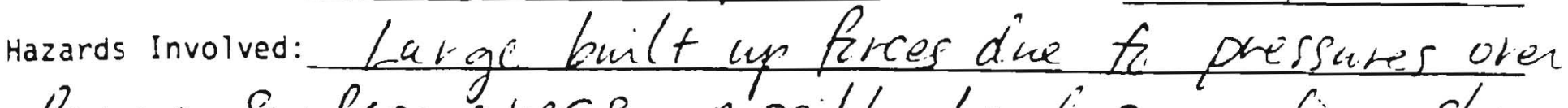
lurge Surfore areas, pissible head separahion, shrap-

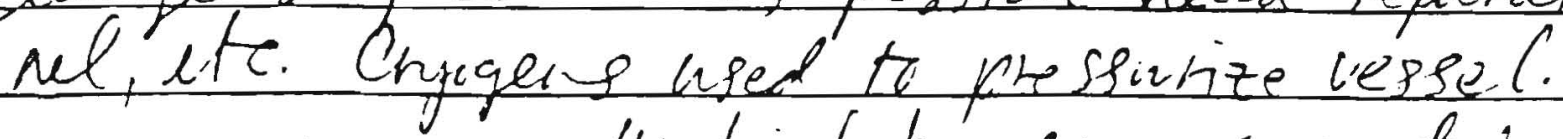

Safety precautions Taken: Lli. high buy area liacuated uside frem tegt perscumel in pit protected bu conerete well. Relicf cialves cin piping.

special conditions or Requirements: Llfe reivete carvere to nunitir pressiche, The laculem hec as will mat be attueled at this tinie.

Test Coordinator: Dept/Date: RP/Dф 25Juneq

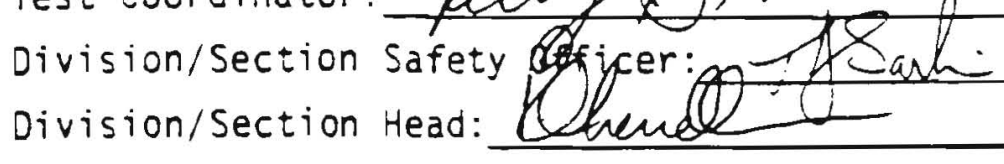
Dept/Date: RDES/4 25 une9l Dept/Date: RDO feme 26,91 Results: Less than $1 / 4$ psi drop in 30 minutes of 30 psig starting pressure.

Date: 25 fune 91 


\section{Pressure Relief Appendices}

Pressure Relief Calculation Summary......................................2

Summary of Argon Sources Investigated................................3

Simplified Cryostat Venting Arrangement.............................. 4

Determine ECN Relieving Flow at S.S. Op'n Conditions...........5

Determine ECN Max. Average Module Filling

Temperature with Given Relieving Capacity..........................15

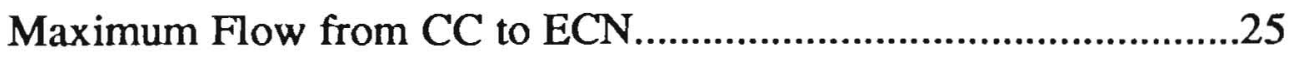

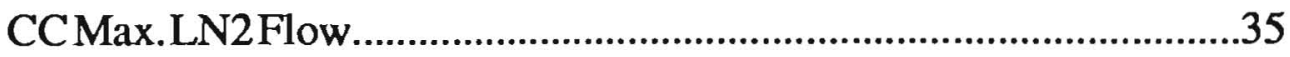

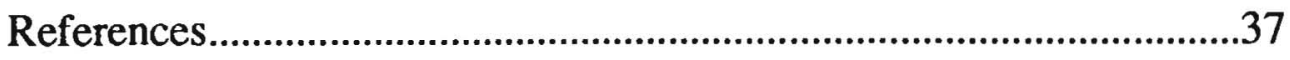

Vacuum Vessel Engineering Note.................................ppended 


\section{Pressure Relief Calculation Summary}

\section{Condition}

1. Fire

2. Insulating Vacuum Loss

3. Condenser Coil Rupture

4. Filling While Modules are Warm
Req'd Flow Capacity (scfm air)

450 (ref. EN-6)

29 (ref. EN-6)

443 (ref. EN-263)

$1572 @ 155 K^{*}$

The total relief capacity for both rupture disk and relief valve evaluated at $96 \mathrm{~K}$ with $3477 \mathrm{lb} / \mathrm{hr}$ saturated GN2 flowing simultaneously through the vent piping is 1604 scfm air.

* The worst case situation is when the CC vessel is full and accidentally discharges LAr through the fill/drain line into a warm ECN. This flowrate rate matches the relief system capacity at a relief device inlet temperature of $155 \mathrm{~K}$, both protective equipment and procedures will ensure that no attempt will be made to fill the cryostat above this temperature. 


\section{Summary of Argon Sources Investigated}

\begin{tabular}{|c|c|c|c|c|}
\hline Source & $\begin{array}{c}\text { Source Relief } \\
\text { Pressure } \\
\text { (psig) }\end{array}$ & $\begin{array}{c}\text { maximum } \\
\text { driving } \\
\text { pressure (psi) }\end{array}$ & $\begin{array}{c}\text { maximum } \\
\text { flowrate } \\
\text { (gpm) }\end{array}$ & $\begin{array}{c}\text { maximum } \\
\text { module } \\
\text { temp. (K) }\end{array}$ \\
\hline LAr Dewar & 20 & 3.21 & 12.3 & 290 \\
\hline CC cryostat & 13 & 3.11 & 18.4 & 155 \\
\hline any & - & - & 24.1 & 96 \\
\hline
\end{tabular}

Note: All values based on flow through both relief valve and rupture disk. 


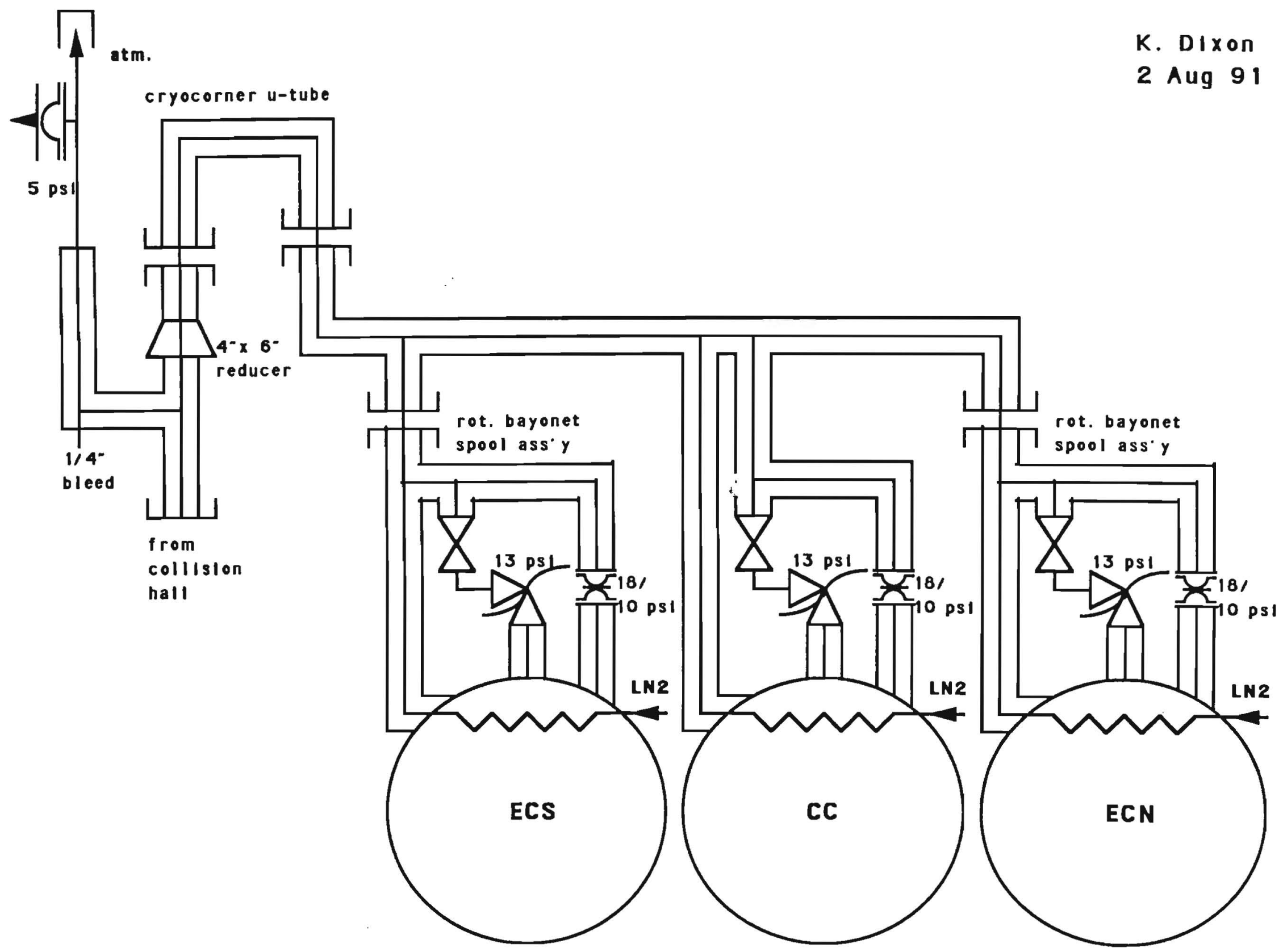

Simplified Cryostat Venting Arrangement 


\section{Determine ECN Relieving Flow at Steady State Operating Conditions}

The following calculations arranged in a spreadsheet format derive the flowrate from both ECN relieving devices. In this case it is assumed that the ECN is full of liquid argon and it is in its steady state cooling mode. One of the other cryostats is assumed to be cooling down while the other is being filled with LAr.

Other assumptions in this analysis include:

1. Pressure in the cryostat is 19.75 psig $(1.16 \mathrm{X}(\mathrm{MAWP}+\mathrm{FV}))$.

2. Gaseous Nitrogen is concurrently flowing in the vent piping at a rate of $3477 \mathrm{lb} / \mathrm{hr}$. This is derived from $0.3 \mathrm{gpm}$ required for ECN steady state conditions, $4 \mathrm{gpm}$ required for cooldown ( $\max$.), and $5 \mathrm{gpm}$ required for filling with LAr (max.)

3. Mixture mass flows are at their maximum at the junction of the relief device outlets on the ECN (GN2 mass flow actually increases gradually at junctions toward the ECS and there is a short segment of piping between the GAr outlets and the condenser exhaust).

4. The temperature in the vent piping is negligible since a large majority of this piping is insulated.

5. All flows are treated as incompressible (max. Mach No. $<0.3)$.

6. The temperature of the GN2 prior to mixing in the vent manifold is 84 $\mathrm{K}$, saturated property at $2 \mathrm{~atm}$.

7. Flow equations apply to weight-averaged mixture densities and viscosities.

For further information on the formulas used and the rationale for the calculations performed in each section, refer to EN-321, "Cryostat Filling Limitations for Proposed Ar Dewar Pressure Increase". 


\section{Explanation of Methodology}

The basic purpose of the spreadsheet was to provide a complete model so that the maximum flow of argon to the reliefs (line 3 ) could be determined. The bulk temperature of the modules (line 2) was set at $96 \mathrm{~K}$, the saturation temperature of argon at the cryostat pressure of $19.75 \mathrm{psig.} \mathrm{A}$ number was then picked as a guess for the maximum argon flow (line 3 ). Then a number was picked as a guess for the percent of mass flow to the relief valve (line 18). From these two numbers, the actual flow through the relief valve (line 98) could be determined. The pressure drops across the inlet and outlets of the relief devices were then calculated. This allowed the calculation of the pressure drops across the relief valve and rupture disk.

The section " $\Delta \mathrm{P}$ Across Relief Valve" calculated the maximum theoretical flow of argon through the relief valve (line 108). Then the theoretical percent of relief flow (line 109) was calculated based on the theoretical relief valve flow divided by the total flow through both the relief valve and rupture disk. The number guessed for the percent of flow to the relief valve (line 18) was then adjusted by iteration until it was close to, but not greater than, the theoretical value (line 109). At this point, the relief valve was operating near its full capacity, which could be checked by noting that the actual flow (line 98) was close to the maximum theoretical flow (line 108).

The next step was to examine the section " $\Delta \mathrm{P}$ Across Rupture Disk". All of the total argon flow not going though the relief valve would be flowing through the rupture disk. In order to insure that the rupture disk could handle the flow, the maximum theoretical rupture disk flow (line 181) was calculated, and compared to the actual flow (line 182). The actual flow had to be under the maximum theoretical flow, but should be close to the maximum value to obtain the highest total flow. In the case that the actual flow was calculated to be higher than the maximum theoretical flow, the total flow rate was lowered. Using the new total flow, the first set of iterations was repeated to match the percent of flow to the relief valve, and the rupture disk flow was compared again. The total flow was lowered through iteration until an acceptable value was found. 
Note that the sections on pressure drops were only needed to calculate inlet and outlet pressures for the relief valve and rupture disk. Other sections calculated the changes in various properties of the argon at certain points. The maximum flow of nitrogen from the condensers was also accounted for, since it had an effect on the pressure drops of the outlets of the relief devices.

\section{Conclusions}

The total mass flow capacity of argon through the reliefs at saturated conditions is given on line 13 and the air equivalent flow@STP is printed on line 15. The percentage of these flows going through the relief valve is shown on line 18. This relief valve flow is calculated to be $760 \mathrm{scfm}$ air; this value is much higher than the usual failure scenarios given in the summary page.

A summation of the pressure drops appears on lines 156 through 165 . Since the pilot senses pressure near the rupture disk inlet, there was concern that the inlet pressure drop to the rupture disk (line 161) might be large enough that the pressure being sensed would drop below the relief valve's reset pressure, closing the relief valve. An investigation into this possibility concluded that the it could be avoided by increasing the blowdown of the relief valve to its maximum of $18 \%$ of the set pressure. Taking into account a maximum error of $3 \%$ (ref. Anderson Greenwood), the worst case blowdown would be $(18 \%-3 \%) \times(13$ psi set pressure $)=$ $1.95 \mathrm{psi}$, which results in a margin of about 1 psi between the relief valve reset pressure ( $13 \mathrm{psig}-1.95 \mathrm{psi}=11.05 \mathrm{psig}=25.746 \mathrm{psia})$ and the lowest pressure at the rupture disk inlet (26.719 psia based on the spreadsheet pressure drops). This margin was accepted by the safety committee, and the relief valve blowdowns on all three cryostats are set at the maximum. 


\section{Maximum ECN Rellef Flow Calculation 10/10/91}

\begin{tabular}{|c|c|c|c|c|}
\hline & \begin{tabular}{|c|} 
A \\
\end{tabular} & $\mathbf{B}$ & $\mathbf{C}$ & $\mathbf{D}$ \\
\hline 1 & Conversion of Liquid to Gas at Module Temp. & & & Units \\
\hline 2 & $>$ Bulk Temp. of Modules & 96 & 96 & $K$ \\
\hline 3 & $>^{*}$ Max. Liquid Equivalent Flow of Argon to Reliefs & 24.1 & 24.1 & $\mathrm{gpm}$ \\
\hline 4 & $>$ Pressure in Cryostat & 19.75 & 19.75 & psig \\
\hline 5 & Pressure in Cryostat (conv.) & $=(B 4 / 14.696+1) * 1.01325$ & 2.375 & bars \\
\hline 6 & $>\operatorname{IAr}$ Density @ 2.2 bars & 1.342421 & 1.342 & $\mathrm{~g} / \mathrm{cc}$ \\
\hline 7 & $>$ IAr Density @ 2.4 bars & 1.335861 & 1.336 & $g / c c$ \\
\hline 8 & Ar Density@ 2.375 bars & $=(\mathrm{B} 5-2.2) / 0.2^{*}(\mathrm{~B} 7-\mathrm{B} 6)+\mathrm{B} 6$ & 1.337 & $g / c c$ \\
\hline 9 & $>$ gAr Density @ 2.2 bars & 11.77 & 11.77 & $\mathrm{mg} / \mathrm{cc}$ \\
\hline 10 & $>$ gAr Density@ 2.4 bars & 12.75 & 12.75 & $\mathrm{mg} / \mathrm{cc}$ \\
\hline 11 & gAr Density @ 2.375 \& Temp. & $=(B 5-2.2) / 0.2^{*}(\mathrm{~B} 10-\mathrm{B} 9)+\mathrm{Bg}$ & 12.627 & $\mathrm{mg} / \mathrm{cc}$ \\
\hline 12 & gAr Density@2.375 bars (conv.) & $=\mathrm{B} 11 / 1000 * 62.428$ & 0.788 & $1 \mathrm{bm} / \mathrm{ft}^{\wedge} 3$ \\
\hline 13 & Maximum Flow of Argon Gas & $=\mathrm{B} 3 * \mathrm{~B} 8 / \mathrm{B} 11 * 1000$ & 2551 & gpm \\
\hline 14 & Maximum Flow of Argon Gas (conv.) & $=\mathrm{B} 13^{*} 0.13368$ & 341 & $\mathrm{cim}$ \\
\hline 15 & Air Equivalent Flow @STP & $=6.32^{*} \mathrm{~B} 17^{*} 356 / \mathrm{B} 16^{*} \mathrm{SQRT}\left(\mathrm{B} 99 /\left(520^{*} \mathrm{~B} 177^{*} 28.97\right)\right)$ & 1604 & scfm air \\
\hline 16 & Specific Heat Constant, $C_{\text {, for }} \mathrm{Ar}$ & $=520^{*} \operatorname{SQRT}\left(\mathrm{B} 168^{*}(2 /(\mathrm{B} 168+1))^{\wedge}((\mathrm{B} 168+1) /(\mathrm{B} 168-1))\right)$ & 383 & \\
\hline 17 & Max. Mass Flow of Argon Gas & $=\mathrm{B} 14^{*} \mathrm{~B} 12^{*} 60$ & 16130 & $\mathrm{~b} \mathrm{~m} / \mathrm{hr}$ \\
\hline 18 & "> Percent of Mass Flow to Relief Valve & 0.472 & 0.472 & \\
\hline \multicolumn{5}{|c|}{19} \\
\hline 20 & $\Delta \mathrm{P}$ Across Relief Valve Inlet & & & Units \\
\hline 21 & $>$ Inner Pipe Diameter & 0.206 & 0.206 & f $t$ \\
\hline 22 & Inner Pipe Diameter (conv.) & $=\mathrm{B} 21 * 12$ & 2.472 & in \\
\hline 23 & $>$ Equivalent Length & 38 & 38 & $f t$ \\
\hline 24 & Ar Gas Density @ 2.375 \& Temp. & $=(\mathrm{B} 5-2.2) / 0.2^{*}(\mathrm{~B} 10-\mathrm{B} 9)+\mathrm{B} 9$ & 12.627 & $\mathrm{mg} / \mathrm{cc}$ \\
\hline 25 & Ar Gas Density @ 2.375 bars (conv.) & $=\mathrm{B} 24 / 1000^{*} 62.428$ & 0.788 & $1 \mathrm{bm} / \mathrm{ft}^{\wedge} 3$ \\
\hline 26 & $>$ gAr Viscosity @ 2.4 bars \& Temp. & 0.0000803 & 0.0000803 & $\mathrm{~g} / \mathrm{cm}-\mathrm{s}$ \\
\hline 27 & gAr Viscosity @ 2.4 bars (conv.) & $=\mathrm{B} 26 * 100$ & 0.00803 & centipoise \\
\hline 28 & Max. Mass Flow to Relief Valve & $=\mathrm{B} 17^{*} \mathrm{~B} 18$ & 7614 & $\mathrm{~b} \mathrm{~m} / \mathrm{hr}$ \\
\hline 29 & Reynolds Number & $=6.31 * \mathrm{~B} 28 /(\mathrm{B} 22 * \mathrm{~B} 27)$ & 2420000 & \\
\hline 30 & $>$ Relative Roughness $(\theta / D)$ & 0.0007 & 0.0007 & \\
\hline 31 & Friction Factor Guess & $=0.25^{\star}\left(\mathrm{LOG}\left(\mathrm{B} 30 / 3.7+5.74 /\left(\mathrm{B} 29^{\wedge} 0.9\right)\right)\right)^{\wedge}-2$ & 0.0183 & \\
\hline 32 & Friction Factor & $=0.25^{\star}\left(\mathrm{LOG}\left(\mathrm{B} 30 / 3.7+2.51 /\left(\mathrm{B} 29^{*} \mathrm{~B} 31^{\wedge} 0.5\right)\right)\right)^{\wedge}-2$ & 0.0182 & \\
\hline 33 & Pressure Drop & $=0.00000336^{*} \mathrm{~B} 32^{*} \mathrm{~B} 23^{*}\left(\mathrm{~B} 28^{\wedge} 2\right) / \mathrm{B} 25 /\left(\mathrm{B} 22^{\wedge} 5\right)$ & 1.852 & psi \\
\hline 34 & & & & \\
\hline
\end{tabular}




\section{Maximum ECN Relief Flow Calculation 10/10/91}

\begin{tabular}{|c|c|c|c|c|}
\hline & A & $\mathbf{B}$ & $\mathbf{C}$ & $\mathbf{D}$ \\
\hline 35 & $\Delta \mathrm{P}$ Across Rupture Disk Inlet & & & Units \\
\hline 36 & Inner Pipe Diameter (conv.) & $-B 37 / 12$ & 0.172 & $f$ \\
\hline 37 & $>$ Inner Pipe Diameter & 2.067 & 2.067 & in \\
\hline 38 & $>$ Equivalent Length & 49 & 49 & $f t$ \\
\hline 39 & gAr Density @ 2.375 \& Temp. & $=(B 5-2.2) / 0.2^{*}(B 10-B 9)+B 9$ & 12.627 & $\mathrm{mg} / \mathrm{cc}$ \\
\hline 40 & gAr Density @ 2.375 bars (conv.) & $=B 39 / 1000 * 62.428$ & 0.788 & $\mathrm{Ibm} / \mathrm{ft} \wedge 3$ \\
\hline 41 & gAr Viscosity @ 2.4 bars & $=\mathrm{B} 26$ & 0.0000803 & $\mathrm{~g} / \mathrm{cm}-\mathrm{s}$ \\
\hline 42 & gAr Viscosity@ 2.4 bars (conv.) & $=\mathrm{B} 41 \cdot 100$ & 0.00803 & centipoise \\
\hline 43 & Max. Mass Flow to Rupture Disk & $=\mathrm{B} 17^{*}(1-\mathrm{B} 18)$ & 8517 & $\mathrm{lb} \mathrm{m} / \mathrm{hr}$ \\
\hline 44 & Reynolds Number & $=6.31^{\circ} \mathrm{B} 43 /\left(\mathrm{B} 37^{\circ} \mathrm{B} 42\right)$ & 3240000 & \\
\hline 45 & $>$ Relative Roughness (e/D) & 0.0009 & 0.0009 & \\
\hline 46 & Friction Factor Guess & $=0.25^{*}\left(\mathrm{LOG}\left(\mathrm{B} 45 / 3.7+5.74 /\left(\mathrm{B} 44^{\wedge} 0.9\right)\right)\right)^{\wedge}-2$ & 0.0193 & \\
\hline 47 & Friction Factor & $=0.25^{*}\left(\mathrm{LOG}\left(\mathrm{B} 45 / 3.7+2.51 /\left(\mathrm{B} 44^{*} \mathrm{~B} 46^{\wedge} 0.5\right)\right)\right)^{\wedge}-2$ & 0.0192 & \\
\hline 48 & Pressure Drop & $=0.00000336^{\star} \mathrm{B} 47^{*} \mathrm{~B} 38^{*}\left(\mathrm{~B} 43^{\wedge} 2\right) / \mathrm{B} 40 /\left(\mathrm{B} 37^{\wedge} 5\right)$ & 7.728 & psi \\
\hline \multicolumn{5}{|c|}{51} \\
\hline 52 & $\Delta \mathrm{P}$ Across Relief Valve Outlet & & & Units \\
\hline 53 & Inner Pipe Diameter (conv.) & $=\mathrm{B} 54 / 12$ & 0.272 & $f t$ \\
\hline 54 & $>$ Inner Pipe Diameter & 3.26 & 3.26 & in \\
\hline 55 & $>$ Equivalent Length & 51 & 51 & $f i$ \\
\hline 56 & $>$ gAr Density @ 2.0 bar \& Temp. & 10.55 & 10.55 & $\mathrm{mg} / \mathrm{cc}$ \\
\hline 57 & gAr Density @ 2.0 bar (conv.) & $=\mathrm{B} 56 / 1000^{*} 62.428$ & 0.659 & $1 \mathrm{bm} / \mathrm{ft}^{\wedge} 3$ \\
\hline 58 & $>$ gAr Viscosity @ 2.0 bar \& Temp. & 0.0000798 & 0.0000798 & $\mathrm{~g} / \mathrm{cm}-\mathrm{s}$ \\
\hline 59 & gAr Viscosity @ 2.0 bar (conv.) & $=\mathrm{B} 58 * 100$ & 0.00798 & centipoise \\
\hline 60 & Max. Mass Flow to Relief Valve & $=\mathrm{B} 28$ & 7614 & $\mathrm{lbm} / \mathrm{hr}$ \\
\hline 61 & Reynolds Number & $=6.31^{*} \mathrm{~B} 60 /\left(\mathrm{B} 54^{*} \mathrm{~B} 59\right)$ & 1850000 & \\
\hline 62 & $>$ Relative Roughness $(\theta / D)$ & 0.00055 & 0.00055 & \\
\hline 63 & Friction Factor Guess & $=0.25^{*}\left(\mathrm{LOG}\left(\mathrm{B} 62 / 3.7+5.74 /\left(\mathrm{B} 61^{\wedge} 0.9\right)\right)\right)^{\wedge}-2$ & 0.0174 & \\
\hline 64 & Friction Factor & $=0.25^{*}\left(\mathrm{LOG}\left(\mathrm{B} 62 / 3.7+2.51 /\left(\mathrm{B} 61^{*} \mathrm{~B} 63^{\wedge} 0.5\right)\right)\right)^{\wedge}-2$ & 0.0173 & \\
\hline 65 & Pressure Drop & $=0.00000336^{*} \mathrm{~B} 64^{*} \mathrm{~B} 55^{*}\left(\mathrm{~B} 60^{\wedge} 2\right) / \mathrm{B} 57 /\left(\mathrm{B} 54^{\wedge} 5\right)$ & 0.71 & psi \\
\hline 66 & & & & \\
\hline
\end{tabular}




\begin{tabular}{|c|c|c|c|c|}
\hline & A & $\mathbf{B}$ & $\mathbf{C}$ & D \\
\hline 67 & $\triangle \mathrm{P}$ Across Rupture Disk Outlet & & & Units \\
\hline 68 & Inner Pipe Diameter (conv.) & $-\mathrm{B} 69 / 12$ & 0.18 & 8 \\
\hline 69 & $>$ Inner Pipe Diameter & 2.157 & 2.157 & in \\
\hline 70 & $>$ Equivalent Length & 1.75 & 1.75 & $f t$ \\
\hline 71 & gAr Density @ 2.0 bar \& Temp. & $=\mathrm{B} 56$ & 10.55 & $\mathrm{mg} / \mathrm{cc}$ \\
\hline 72 & gAr Density @ 2.0 bar (conv.) & $=B 71 / 1000^{*} 62.428$ & 0.659 & $1 \mathrm{bm} / \mathrm{ft} \wedge 3$ \\
\hline 73 & gAr Viscosity @ 2.0 bar \& Temp. & $=\mathrm{B} 58$ & 0.0000798 & $\mathrm{~g} / \mathrm{cm}-\mathrm{s}$ \\
\hline 74 & gAr Viscosity@2.0 bar (conv.) & $=\mathrm{B} 73 * 100$ & 0.00798 & centipoise \\
\hline 75 & Max. Mass Flow to Rupture Disk & $=\mathrm{B} 43$ & 8517 & $1 \mathrm{bm} / \mathrm{hr}$ \\
\hline 76 & Reynolds Number & $=6.31^{\circ} \mathrm{B} 75 /\left(\mathrm{B} 69^{\circ} \mathrm{B} 74\right)$ & 3120000 & \\
\hline 77 & $>$ Relative Roughness (e/D) & 0.0009 & 0.0009 & \\
\hline 78 & Friction Factor Guess & $-0.25^{\star}\left(\mathrm{LOG}\left(\mathrm{B} 77 / 3.7+5.74 /\left(\mathrm{B} 76^{\wedge} 0.9\right)\right)\right)^{\wedge}-2$ & 0.0193 & \\
\hline 79 & Friction Factor & $=0.25^{*}\left(\mathrm{LOG}\left(\mathrm{B} 77 / 3.7+2.51 /\left(\mathrm{B} 76^{*} \mathrm{~B} 78^{\wedge} 0.5\right)\right)\right)^{\wedge}-2$ & 0.0193 & \\
\hline 80 & Pressure Drop & $=0.00000336^{\star} \mathrm{B} 79^{*} \mathrm{~B} 70^{*}\left(\mathrm{~B} 75^{\wedge} 2\right) / \mathrm{B} 72 /\left(\mathrm{B} 69^{\wedge} 5\right)$ & 0.267 & psi \\
\hline \multicolumn{5}{|c|}{81 (2) } \\
\hline 82 & Change in Gas at Common Outlet to Outside & & & Units \\
\hline 83 & Pressure in Cryostat & $=\mathrm{B} 4$ & 19.75 & psig \\
\hline 84 & Pressure in Cryostat (conv.) & $=(B 83 / 14.696+1) * 1.01325$ & 2.375 & bars \\
\hline 85 & gAr Density @ 2.2 bars & -B9 & 11.77 & $m g / c c$ \\
\hline 86 & gAr Density @ 2.4 bars & $=\mathrm{B} 10$ & 12.75 & $\mathrm{mg} / \mathrm{cc}$ \\
\hline 87 & gAr Density@ 2.375 \& Temp. & $=(\mathrm{B} 84-2.2) / 0.2^{*}(\mathrm{~B} 86-\mathrm{B} 85)+\mathrm{B} 85$ & 12.627 & $\mathrm{mg} / \mathrm{cc}$ \\
\hline 88 & Temp. at Common Outlet & $=\left(\mathrm{B} 2^{*} \mathrm{~B} 17+84^{*} \mathrm{~B} 189\right) /(\mathrm{B} 17+\mathrm{B} 189)$ & 94 & $\mathrm{~K}$ \\
\hline 89 & $>$ Pressure to Calculate Density & 1.5 & 1.5 & bars \\
\hline 90 & * gAr Density @ 1.5 bars \& New Temp. & 7.99 & 7.99 & $\mathrm{mg} / \mathrm{cc}$ \\
\hline 91 & "> gAr Viscosity @ 1.5 bars \& New Temp. & 0.000078 & 0.000078 & $\mathrm{~g} / \mathrm{cm}-\mathrm{s}$ \\
\hline 92 & gAr Viscosity @ 1.5 bar (conv.) & $=\mathrm{B} 91 * 100$ & 0.0078 & centipoise \\
\hline 93 & & & & \\
\hline
\end{tabular}




\section{Maximum ECN Rellef Flow Calculation 10/10/91}

\begin{tabular}{|c|c|c|c|c|}
\hline & A & $\mathbf{B}$ & C & $\mathbf{D}$ \\
\hline 94 & $\Delta \mathrm{P}$ Across Relief Valve & & & Units \\
\hline 95 & $>$ Critical Ratio (Pcr/P1) for Argon & 0.487 & 0.487 & \\
\hline 96 & $>$ Specific Heat Ratio (k) for Argon & $=\mathrm{B} 168$ & 1.75 & \\
\hline 97 & $>$ Area of $2^{n} \times 3^{n}$ Relief Valve & 2.29 & 2.29 & $\operatorname{in}^{\wedge} 2$ \\
\hline 98 & Flow Through Relief Valve & $=\mathrm{B} 28$ & 7614 & $\mathrm{lbm} / \mathrm{hr}$ \\
\hline 99 & Flowing Temperature & $=1.8 * \mathrm{~B} 2$ & 173 & $\operatorname{deg} R$ \\
\hline 100 & $>$ Compressibility Factor & 1 & 1 & \\
\hline 101 & $>$ Nozzle Coefficient for type 93T & 0.939 & 0.939 & \\
\hline 102 & Flowing Inlet Pressure (P1) & $=\mathrm{B} 4+14.696-\mathrm{B} 33$ & 32.59 & psia \\
\hline 103 & $>$ Molecular Weight of Argon & 39.948 & 39.95 & $\mathrm{~g} / \mathrm{mol}$ \\
\hline 104 & Critical Pressure (Pcr) & $=\mathrm{B} 95^{*} \mathrm{~B} 102$ & 15.87 & psia \\
\hline 105 & Outlet Pressure (P2) (using delta p's) & $=14.696+\mathrm{B} 154+\mathrm{B} 133+\mathrm{B} 65$ & 25.96 & psia \\
\hline 106 & Pressure Ratio (P2*/P1) & $=\left(\mathrm{B} 102-0.55^{*}\left((\mathrm{~B} 102-\mathrm{B} 105)^{\wedge} 0.98\right)\right) / \mathrm{B} 102$ & 0.892 & \\
\hline 107 & Theoretical Factor (F") (using P2) & $=\mathrm{SQRT}\left((\mathrm{B} 96 /(\mathrm{B} 96-1))^{\star}\left(\mathrm{B} 106^{\wedge}(2 / \mathrm{B} 96)-\mathrm{B} 106^{\wedge}((\mathrm{B} 96+1) / \mathrm{B} 96)\right)\right)$ & 0.313 & \\
\hline 108 & Max. Theoretical Relief Flow (using $\mathrm{F}^{*}$ ) & $=735^{*} \mathrm{~B} 97^{*} \mathrm{~B} 101^{*} \mathrm{~B} 102^{*} \mathrm{~B} 107^{*} \mathrm{SQRT}(\mathrm{B} 103 / \mathrm{B} 99 / \mathrm{B} 100)$ & 7740 & $1 \mathrm{bm} / \mathrm{hr}$ \\
\hline 109 & Theoretical Percent of Relief Flow & $=\mathrm{B} 108 / \mathrm{B} 17$ & 0.4799 & \\
\hline 110 & Pressure Drop Across Relief Valve & $=\mathrm{B} 102-\mathrm{B} 105$ & 6.633 & psi \\
\hline 111 & & & & \\
\hline
\end{tabular}




\section{Maximum ECN Rellef Flow Calculation 10/10/91}

\begin{tabular}{|c|c|c|c|c|}
\hline & A & $\mathbf{B}$ & C & D \\
\hline 112 & $\triangle \mathrm{P}$ Across Common Outlet to Cryocorner & & & Units \\
\hline 113 & > Inner Pipe Diameter & 0.355 & 0.355 & $f t$ \\
\hline 114 & Inner Pipe Diameter (conv.) & $=\mathrm{B} 113^{*} 12$ & 4.26 & in \\
\hline 115 & $>$ Equivalent Length & 273 & 273 & $f t$ \\
\hline 116 & gAr Density @1.5 bar \& New Temp. & $=890$ & 7.99 & $\mathrm{mg} / \mathrm{cc}$ \\
\hline 117 & gAr Density @ 1.5 bar (conv.) & $=B 116 / 1000 * 62.428$ & 0.499 & $1 \mathrm{bm} / \mathrm{ft}^{\wedge} 3$ \\
\hline 118 & "> gN2 Gas Density @ 1.5 bar \& New Temp. & 5.653 & 5.653 & $\mathrm{mg} / \mathrm{cc}$ \\
\hline 119 & gN2 Gas Density @ 1.5 bar (conv.) & $=B 118 / 1000^{*} 62.428$ & 0.353 & $1 \mathrm{bm} / \mathrm{ft}^{\wedge} 3$ \\
\hline 120 & Gas Mixture Density @1.5 bar & $=\left(\mathrm{B} 17^{*} \mathrm{~B} 117+\mathrm{B} 127^{*} \mathrm{~B} 119\right) / \mathrm{B} 128$ & 0.473 & $1 \mathrm{bm} / \mathrm{ft}^{\wedge} 3$ \\
\hline 121 & gAr Viscosity @ 1.5 bar \& New Temp. & $=\mathrm{B} 91$ & 0.000078 & $\mathrm{~g} / \mathrm{cm}-\mathrm{s}$ \\
\hline 122 & gAr Viscosity@1.5 bar (conv.) & $-B 121 * 100$ & 0.0078 & centipoise \\
\hline 123 & "> gN2 Viscosity @ 1.5 bar \& New Temp. & 0.0000643 & 0.0000643 & $\mathrm{~g} / \mathrm{cm}-\mathrm{s}$ \\
\hline 124 & gN2 Viscosity@1.5 bar (conv.) & $=\mathrm{B} 123^{*} 100$ & 0.00643 & centipoise \\
\hline 125 & Mixture Viscosity @1.5 bar & $=\left(\mathrm{B} 17^{*} \mathrm{~B} 122+\mathrm{B} 127^{*} \mathrm{~B} 124\right) / \mathrm{B} 128$ & 0.007557 & centipoise \\
\hline 126 & Max. Mass Flow of Argon Gas & $=\mathrm{B} 17$ & 16130 & $\mathrm{lbm} / \mathrm{hr}$ \\
\hline 127 & Max. Flow of Nitrogen Gas & $=\mathrm{B} 189$ & 3477 & $16 \mathrm{~m} / \mathrm{hr}$ \\
\hline 128 & Mass Flow of Mixture & $=\mathrm{B} 126+\mathrm{B} 127$ & 19608 & $\mathrm{lbm} / \mathrm{hr}$ \\
\hline 129 & Reynolds Number & $=6.31^{*} \mathrm{~B} 128 /\left(\mathrm{B} 114^{*} \mathrm{~B} 125\right)$ & 3840000 & \\
\hline 130 & $>$ Relative Roughness (e/D) & 0.0004 & 0.0004 & \\
\hline 131 & Friction Factor Guess & $-0.25^{\star}\left(\mathrm{LOG}\left(\mathrm{B} 130 / 3.7+5.74 /\left(\mathrm{B} 129^{\wedge} 0.9\right)\right)\right)^{\wedge}-2$ & 0.0161 & \\
\hline 132 & Friction Factor & $=0.25^{\star}\left(\mathrm{LOG}\left(\mathrm{B} 130 / 3.7+2.51 /\left(\mathrm{B} 129^{*} \mathrm{~B} 131^{\wedge} 0.5\right)\right)\right)^{\wedge}-2$ & 0.0161 & \\
\hline 133 & Pressure Drop & $=0.00000336^{*} \mathrm{~B} 132^{*} \mathrm{~B} 115^{*}\left(\mathrm{~B} 128^{\wedge} 2\right) / \mathrm{B} 120 /\left(\mathrm{B} 114^{\wedge} 5\right)$ & 8.534 & psi \\
\hline 134 & & & & \\
\hline
\end{tabular}




\section{Maximum ECN Rellef_Flow Calculation_ 10/10/91}

\begin{tabular}{|c|c|c|c|c|}
\hline & A & $\mathbf{B}$ & C & D \\
\hline 135 & $\Delta \mathrm{P}$ from Cryocorner to Outside & & & Units \\
\hline 136 & $>$ Inner Pipe Diameter & 0.53 & 0.53 & $f t$ \\
\hline 137 & Inner Pipe Diameter (conv.) & $=\mathrm{B} 136 * 12$ & 6.36 & in \\
\hline 138 & $>$ Equivalent Length & 516 & 516 & f t \\
\hline 139 & gAr Gas Density @ 1.5 bar \& New Temp. & $=\mathrm{B} 90$ & 7.99 & $\mathrm{mg} / \mathrm{cc}$ \\
\hline 140 & gAr Gas Density@ @ 1.5 bar (conv.) & $=\mathrm{B} 139 / 1000^{\circ} 62.428$ & 0.499 & $16 \mathrm{~m} / \mathrm{ft}^{\wedge} 3$ \\
\hline 141 & gN2 Gas Density @ 1.5 bar \& New Temp. & $=\mathrm{B} 118$ & 5.653 & $\mathrm{mg} / \mathrm{cc}$ \\
\hline 142 & gN2 Gas Density @ 1.5 bar (conv.) & $=\mathrm{B} 141 / 1000^{*} 62.428$ & 0.353 & $1 \mathrm{bm} / \mathrm{ft} \wedge 3$ \\
\hline 143 & Gas Mixture Density @1.5 bar & $=\left(\mathrm{B} 17^{*} \mathrm{~B} 140+\mathrm{B} 189^{*} \mathrm{~B} 142\right) / \mathrm{B} 149$ & 0.473 & $\mathrm{lbm} / \mathrm{ft}^{\wedge} 3$ \\
\hline 144 & gAr Viscosity @ 1.5 bar \& New Temp. & $=\mathrm{B} 121$ & 0.000078 & $\mathrm{~g} / \mathrm{cm}-\mathrm{s}$ \\
\hline 145 & gAr Viscosity @ 1.5 bar (conv.) & $=\mathrm{B} 144 * 100$ & 0.0078 & centipoise \\
\hline 146 & gN2 Viscosity @ 1.5 bar \& New Temp. & $=\mathrm{B} 123$ & $0.000064 \overline{3}$ & $\mathrm{~g} / \mathrm{cm}-\mathrm{s}$ \\
\hline 147 & gN2 Viscosity@1.5 bar (conv.) & $=\mathrm{B} 146 * 100$ & 0.00643 & centipoise \\
\hline 148 & Gas Mixture Viscosity @1.5 bar & $=\left(\mathrm{B} 17^{*} \mathrm{~B} 145+\mathrm{B} 189^{*} \mathrm{~B} 147\right) / \mathrm{B} 149$ & 0.007557 & centipoise \\
\hline 149 & Max. Mass Flow of Gas Mixture & $=\mathrm{B} 128$ & 19608 & $\mathrm{lbm} / \mathrm{hr}$ \\
\hline 150 & Reynolds Number & $=6.31^{\circ} \mathrm{B} 149 /\left(\mathrm{B} 137^{*} \mathrm{~B} 148\right)$ & 2570000 & \\
\hline 151 & $>$ Relative Roughness (e/D) & 0.00027 & 0.00027 & \\
\hline 152 & Friction Factor Guess & $=0.25^{*}\left(\mathrm{LOG}\left(\mathrm{B} 151 / 3.7+5.74 /\left(\mathrm{B} 150^{\wedge} 0.9\right)\right)\right)^{\wedge}-2$ & 0.015 & \\
\hline 153 & Friction Factor & $=0.25^{*}\left(\mathrm{LOG}\left(\mathrm{B} 151 / 3.7+2.51 /\left(\mathrm{B} 150^{*} \mathrm{~B} 152^{\wedge} 0.5\right) \mathrm{U}\right)^{\wedge}-2\right.$ & 0.0149 & \\
\hline 154 & Pressure Drop & $=0.00000336^{*} \mathrm{~B} 153^{*} \mathrm{~B} 138^{*}\left(\mathrm{~B} 149^{\wedge} 2\right) / \mathrm{B} 143 /\left(\mathrm{B} 137^{\wedge} 5\right)$ & 2.022 & psi \\
\hline 155 & & & & \\
\hline 156 & Summation of Equivalent $\Delta \mathrm{Ps}$ & & & Units \\
\hline 157 & Relief Valve Inlet Pressure Drop & $=\mathrm{B} 33$ & 1.852 & psi \\
\hline 158 & Relief Valve Outlet Pressure Drop & $=\mathrm{B} 65$ & 0.71 & psi \\
\hline 159 & Relief Valve Pressure Drop & $=\mathrm{B} 110$ & 6.633 & psi \\
\hline 160 & Relief Valve/Disk Branch & $=\mathrm{B} 33+\mathrm{B} 65+\mathrm{B} 110$ & 9.194 & psi \\
\hline 161 & Rupture Disk Inlet Pressure Drop & $=\mathrm{B} 48$ & 7.728 & psi \\
\hline 162. & Rupture Disk Outlet Pressure Drop & $=\mathrm{B} 80$ & 0.267 & psi \\
\hline 163 & Rupture Disk Pressure Drop & $=\mathrm{B} 157+\mathrm{B} 158+\mathrm{B} 159-\mathrm{B} 161-\mathrm{B} 162$ & 1.199 & psi \\
\hline 164 & Common Outlet Pressure Drop & $=\mathrm{B} 133$ & 8.534 & psi \\
\hline 165 & Cryocorner to Outside Pressure Drop & $=\mathrm{B} 154$ & 2.022 & psi \\
\hline 166 & & & & \\
\hline
\end{tabular}




\section{Maximum ECN Rellef Flow Calculation 10/10/91}

\begin{tabular}{|c|c|c|c|c|}
\hline & A & $\mathbf{B}$ & $\mathbf{C}$ & $\mathbf{D}$ \\
\hline 167 & $\triangle P$ Across Rupture Disk & & & Units \\
\hline 168 & $>$ Argon Specific Heat Ratio (k) & 1.745 & 1.745 & \\
\hline 169 & Critical Ratio & $=(2 /(B 168+1))^{\wedge}(B 168 /(B 168-1))$ & 0.476 & \\
\hline 170 & $>$ Area of $3^{\text {" Rupture Disk }}$ & $=3.14159^{*}\left(3^{\wedge} 2\right) / 4$ & 7.069 & $\operatorname{in}^{\wedge} 2$ \\
\hline 171 & Flow Through Rupture Disk & $=\mathrm{B} 43$ & 8517 & $1 \mathrm{bm} / \mathrm{hr}$ \\
\hline 172 & Flowing Temperature & $=1.8^{n} \mathrm{~B} 2$ & 173 & $\operatorname{deg} R$ \\
\hline 173 & $>$ ASME Coefficient $(K)$ & 0.62 & 0.62 & \\
\hline 174 & Pressure Ratio (Pe/Po) & $=\mathrm{B} 179 / \mathrm{B} 176$ & 0.955 & \\
\hline 175 & gAr Flow Constant for Subsonic Flow(C1) & $=\operatorname{SQRT}\left(2^{*} 32.2 / 1545^{*}(\mathrm{~B} 168 /(\mathrm{B} 168-1))^{*}\left(\mathrm{~B} 174^{\wedge}(2 / \mathrm{B} 168)-\mathrm{B} 174^{\wedge}((\mathrm{B})\right.\right.$ & 0.042 & \\
\hline 176 & Flowing Inlet Pressure (Po) & $=\mathrm{B} 4+14.696-\mathrm{B} 48$ & 26.72 & psia \\
\hline 177 & $>$ Molecular Weight of Argon & 39.948 & 39.948 & $\mathrm{~g} / \mathrm{mol}$ \\
\hline 178 & Critical Pressure (Pcr) & $=\mathrm{B} 169 * \mathrm{~B} 176$ & 12.73 & psia \\
\hline 179 & Outlet Pressure $(\mathrm{Pe})$. (using delta $\mathrm{p}^{\prime} \mathrm{s}$ ) & $=14.696+\mathrm{B} 154+\mathrm{B} 133+\mathrm{B} 80$ & 25.52 & psia \\
\hline 180 & Pressure Drop Across Rupture Disk & $=\mathrm{B} 176-\mathrm{B} 179$ & 1.199 & psi \\
\hline 181 & Maximum Theoretical Rupture Disk Flow & $=\mathrm{B} 170^{*} \mathrm{~B} 173^{*} \mathrm{~B} 175^{*} \mathrm{~B} 176^{*} \mathrm{SQRT}(\mathrm{B} 177 / \mathrm{B} 172)^{*} 60^{\circ} 60$ & 8595 & $\mathrm{lbm} / \mathrm{hr}$ \\
\hline 182 & Actual Rupture Disk Flow & $=\mathrm{B} 43$ & $85 \overline{17}$ & $16 \mathrm{~m} / \mathrm{hr}$ \\
\hline \multicolumn{5}{|c|}{183 (2) } \\
\hline 184 & Maximum Flow from Condensers & & & units \\
\hline 185 & $>$ Max. Flow of Liquid Nitrogen & 9.3 & 9.3 & gpm \\
\hline 186 & Max. Flow of Liquid Nitrogen (conv.) & $=\mathrm{B} 185 / 7.48$ & 1.24 & $f t^{\wedge} 3 / \mathrm{min}$ \\
\hline 187 & Density of LN2 @ $3.5 \mathrm{~atm}$ & 0.747 & 0.747 & $\mathrm{~g} / \mathrm{Cc}$ \\
\hline 188 & Density of LN2 (conv.) & $=\mathrm{B} 187^{*} 62.4$ & 46.6128 & $1 \mathrm{bm} / \mathrm{ft}^{\wedge} 3$ \\
\hline 189 & Mass Flow of LN2 & $=B 186^{*} \mathrm{~B} 188^{*} 60$ & 3477 & $\mathrm{lb} \mathrm{m} / \mathrm{hr}$ \\
\hline \multicolumn{5}{|c|}{190} \\
\hline 191 & Notes: & & & \\
\hline \multicolumn{5}{|c|}{192 " $>$ indicates that this value must be changed for a } \\
\hline \multicolumn{5}{|c|}{\begin{tabular}{|l|l|l|}
193 & new flowrate \\
194 & \\
\end{tabular}} \\
\hline \multicolumn{5}{|c|}{ reouiring shono for now } \\
\hline \multicolumn{5}{|c|}{195 > indicates variable not requiring change for new } \\
\hline \multirow{2}{*}{\multicolumn{5}{|c|}{$\begin{array}{l}196 \text { flowrates } \\
197\end{array}$}} \\
\hline & & & & \\
\hline \multicolumn{5}{|c|}{198 (conv.) indicates the previous value converted to new } \\
\hline 199 & units & & & \\
\hline
\end{tabular}




\section{Determine ECN Maximum Average Module Filling Temperature with Given Relieving Capacity}

The worst case relieving scenario for any of the cryostats is when one of the adjacent vessels is full of LAr and accidentally discharges through the fill/drain line into a warm cryostat (note that this is a worse case than the storage dewar@20 psig filling a warm cryostat as in EN-321). The worst case overall is when the CC discharges liquid into the ECN. The following calculations given in a spreadsheet format with results listed to the right, determine the maximum module temperature that the ECN can be filled at without exceeding the capacity of the present relieving system. In this case the ECN has no accumulation of liquid argon and no allowance is given for the nitrogen cooling although it is assumed to be flowing in the manifold at ECN for back pressure calculations.

Other assumptions in this analysis include:

1. Pressure in the cryostat is 19.75 psig $(1.16 \mathrm{X}(\mathrm{MAWP}+\mathrm{FV}))$.

2. Gaseous Nitrogen is concurrently flowing in the vent piping at a rate of $1608 \mathrm{lb} / \mathrm{hr}$. This is derived from $0.3 \mathrm{gpm}$ required for CC in its steady state mode and $4 \mathrm{gpm}$ required for the concurrent cooldown (max.) of the ECS vessel.

3. Mixture mass flows are at their maximum at the junction of the relief device outlets on the ECN (GN2 mass flow actually increases gradually at junctions toward the ECS and there is a short segment of piping between the GAr outlets and the condenser exhaust).

4. The temperature in the vent piping is negligible since a large majority of this piping is insulated.

5. All flows are treated as incompressible (max. Mach No. $<0.3$ ).

6. The temperature of the GN2 prior to mixing in the vent manifold is 84 $\mathrm{K}$, saturated property at $2 \mathrm{~atm}$.

7. Flow equations apply to weight-averaged mixture densities and viscosities. 
8. All liquid argon flashes to the bulk module temperature in the cryostat prior to entering the piping.

For further information on the formulas used and the rationale for the calculations performed in each section, refer to EN-321, "Cryostat Filling Limitations for Proposed Ar Dewar Pressure Increase".

\section{Explanation of Methodology}

The basic purpose of the spreadsheet was to provide a complete model so that the maximum bulk temperature of the modules (line 2) could be determined. The maximum flow of argon to the ECN (line 3) was calculated separately and included after the spreadsheet. A number was picked as a guess for the bulk temperature (line 2). Then a number was picked as a guess for the percent of mass flow to the relief valve (line 18). The actual flow through the relief valve (line 98) was determined using the total flow and the percent of flow to the relief valve. The pressure drops across the inlet and outlets of the relief devices were then calculated. This allowed the calculation of the pressure drops across the relief valve and rupture disk. The various properties of argon were taken from tables at the bulk temperature of the modules.

The section " $\Delta \mathrm{P}$ Across Relief Valve" calculated the maximum theoretical flow of argon through the relief valve (line 108). Then the theoretical percent of relief flow (line 109) was calculated based on the theoretical relief valve flow divided by the total flow through both the relief valve and rupture disk. The number guessed for the percent of flow to the relief valve (line 18) was then adjusted by iteration until it was close to, but not greater than, the theoretical value (line 109). At this point, the relief valve was operating near its full capacity, which could be checked by noting that the actual flow (line 98) was close to the maximum theoretical flow (line 108). 
The next step was to examine the section " $\Delta \mathrm{P}$ Across Rupture Disk". All of the total argon flow not going though the relief valve would be flowing through the rupture disk. In order to insure that the rupture disk could handle the flow, the maximum theoretical rupture disk flow (line 181) was calculated, and compared to the actual flow (line 182). The actual flow had to be under the maximum theoretical flow, but should be close to the maximum value to obtain the highest total flow. In the case that the actual flow was calculated to be higher than the maximum theoretical flow, the bulk temperature was lowered. Using the new temperature, the first set of iterations was repeated to match the percent of flow to the relief valve, and the rupture disk flow was compared again. The temperature was lowered through iteration until an acceptable value was found.

Note that the sections on pressure drops were only needed to calculate inlet and outlet pressures for the relief valve and rupture disk. Other sections calculated the changes in various properties of the argon at certain points. Each time the temperature was changed, the values for density and viscosity were changed to reflect the new temperature. The maximum flow of nitrogen from the condensers was also accounted for, since it had an effect on the pressure drops of the outlets of the relief devices.

\section{Conclusions}

Since the average module temperature in which the cryostat can be filled is lower than that calculated in EN-321, then this temperature appears to be the limiting case for the cryostat. Procedural safeguards coupled with locked switches on the output electrical signal to the fill valve PV118A I/P transmitter will be used to limit the filling of this cryostat to periods when the average temperature of the modules is $155 \mathrm{~K}$ or lower. This temperature limitation will probably be lower due to operational concerns and the structural safety of the cryostat modules. 


\begin{tabular}{|c|c|c|c|c|}
\hline & A & $\mathbf{B}$ & C & D \\
\hline 1 & Conversion of Liquid to Gas at Module Temp. & & & Units \\
\hline 2 & "> Bulk Temp. of Modules & 155 & 155 & $\mathrm{~K}$ \\
\hline 3 & $>$ Max. Flow of Liquid Argon to Cryostat & 18.4 & 18.4 & gpm \\
\hline 4 & $>$ Pressure in Cryostat & 19.75 & 19.75 & psig \\
\hline 5 & Pressure in Cryostat (conv.) & $=(B 4 / 14.696+1) * 1.01325$ & 2.375 & bars \\
\hline 6 & $>\operatorname{Ar}$ Density @ 2.2 bars & 1.342421 & 1.34 & $\mathrm{~g} / \mathrm{cc}$ \\
\hline 7 & $>$ Ar Density @ 2.4 bars & 1.335861 & 1.34 & $g / c c$ \\
\hline 8 & IAr Density@ @2.375 bars & $=(\mathrm{B} 5-2.2) / 0.2^{*}(\mathrm{~B} 7-\mathrm{B} 6)+\mathrm{B} 6$ & 1.337 & $g / c c$ \\
\hline 9 & "> gAr Density @ 2.2 bars & 6.927 & 6.927 & $\mathrm{mg} / \mathrm{cc}$ \\
\hline 10 & > gAr Density @ 2.4 bars & 7.566 & 7.566 & $\mathrm{mg} / \mathrm{cc}$ \\
\hline 11 & gAr Density @ 2.375 \& Temp. & $=(\mathrm{B} 5-2.2) / 0.2^{*}(\mathrm{~B} 10-\mathrm{B} 9)+\mathrm{Bg}$ & 7.486 & $\mathrm{mg} / \mathrm{cc}$ \\
\hline 12 & gAr Density@2.375 bars (conv.) & $=B 11 / 1000^{*} 62.428$ & 0.467 & $1 \mathrm{bm} / \mathrm{ft}^{\wedge} 3$ \\
\hline 13 & Maximum Flow of Argon Gas & $=\mathrm{B} 3 * \mathrm{~B} 8 / \mathrm{B} 11 * 1000$ & 3285 & gpm \\
\hline 14 & Maximum Flow of Argon Gas (conv.) & $=B 13^{*} 0.13368$ & 439 & $\mathrm{cfm}$ \\
\hline 15 & Air Equivalent Flow @STP & $=6.32^{*} \mathrm{~B} 17^{*} 356 / \mathrm{B}^{\prime} 6^{*} \mathrm{SQRT}\left(\mathrm{B} 99 /\left(520^{*} \mathrm{~B} 177^{*} 28.97\right)\right)$ & 1572 & sctm air \\
\hline 16 & Specific Heat Constant, C, for Ar & $=520^{\circ} \mathrm{SQRT}\left(\mathrm{B} 168^{*}(2 /(\mathrm{B} 168+1))^{\wedge}((\mathrm{B} 168+1) /(\mathrm{B} 168-1))\right)$ & 379 & \\
\hline 17 & Max. Mass Flow of Argon Gas & $=\mathrm{B} 14^{*} \mathrm{~B} 12 * 60$ & 12315 & $\mathrm{lbm} / \mathrm{hr}$ \\
\hline 18 & " $>$ Percent of Mass Flow to Relief Valve & 0.48 & 0.48 & \\
\hline \multicolumn{5}{|c|}{10} \\
\hline 20 & $\Delta \mathrm{P}$ Across Relief Valve Inlet & & & Units \\
\hline 21 & $>$ Inner Pipe Diameter & 0.206 & 0.206 & $f t$ \\
\hline 22 & Inner Pipe Diameter (conv.) & $=\mathrm{B} 21 * 12$ & 2.472 & in \\
\hline 23 & > Equivalent Length & 38 & 38 & $f t$ \\
\hline 24 & Ar Gas Density @ 2.375 \& Temp. & $=(\mathrm{B} 5-2.2) / 0.2^{*}(\mathrm{~B} 10-\mathrm{B} 9)+\mathrm{B} 9$ & 7.486 & $\mathrm{mg} / \mathrm{cc}$ \\
\hline 25 & Ar Gas Density @ 2.375 bars (conv.) & $=B 24 / 1000^{*} 62.428$ & 0.467 & $\mathrm{lbm} / \mathrm{ft}^{\wedge} 3$ \\
\hline 26 & "> gAr Viscosity @ 2.4 bars \& Temp. & 0.0001261 & 0.0001261 & $\mathrm{~g} / \mathrm{cm} \cdot \mathrm{s}$ \\
\hline 27 & gAr Viscosity @ 2.4 bars (conv.) & $-B 26 * 100$ & 0.01261 & centipoise \\
\hline 28 & Max. Mass Flow to Relief Valve & $=\mathrm{B} 17^{*} \mathrm{~B} 18$ & 5911 & $\mathrm{lbm} / \mathrm{hr}$ \\
\hline 29 & Reynolds Number & $-6.31^{*} \mathrm{~B} 28 /\left(\mathrm{B} 22^{*} \mathrm{~B} 27\right)$ & 1200000 & \\
\hline 30 & $>$ Relative Roughness (e/D) & 0.0007 & 0.0007 & \\
\hline 31 & Friction Factor Guess & $=0.25^{*}\left(\operatorname{LOG}\left(B 30 / 3.7+5.74 /\left(B 29^{\wedge} 0.9\right)\right)\right)^{\wedge}-2$ & 0.0185 & \\
\hline 32 & Friction Factor & $=0.25^{*}\left(\mathrm{LOG}\left(\mathrm{B} 30 / 3.7+2.51 /\left(\mathrm{B} 29^{*} \mathrm{~B} 31^{\wedge} 0.5\right)\right)\right)^{\wedge}-2$ & 0.0184 & \\
\hline 33 & Pressure Drop & $=0.00000336^{*} \mathrm{~B} 32^{*} \mathrm{~B} 23^{*}\left(\mathrm{~B} 28^{\wedge} 2\right) / \mathrm{B} 25 /\left(\mathrm{B} 22^{\wedge} 5\right)$ & 1.9 & psi \\
\hline 34 & & & & \\
\hline
\end{tabular}




\section{Maximum Module Temperature Calculation}

$10 / 17 / 91$

\begin{tabular}{|c|c|c|c|c|}
\hline & A & B & C & $\mathbf{D}$ \\
\hline 35 & $\Delta \mathrm{P}$ Across Rupture Disk Inlet & & & Units \\
\hline 36 & Inner Pipe Diameter (conv.) & $-B 37 / 12$ & 0.172 & $f t$ \\
\hline 37 & $>$ Inner Pipe Diameter & 2.067 & $2 . \overline{067}$ & in \\
\hline 38 & $>$ Equivalent Length & 49 & 49 & $\mathrm{ft}$ \\
\hline 39 & gAr Density @ 2.375 \& Temp. & $=(B 5-2.2) / 0.2^{*}(B 10-B 9)+B 9$ & 7.486 & $\mathrm{mg} / \mathrm{cc}$ \\
\hline 40 & gAr Density @ 2.375 bars (conv.) & $-B 39 / 1000^{*} 62.428$ & 0.467 & $\mathrm{lb} \mathrm{m} / \mathrm{ft}^{\wedge} 3$ \\
\hline 41 & gAr Viscosity @ 2.4 bars & $=\mathrm{B} 26$ & 0.0001261 & $\mathrm{~g} / \mathrm{cm} \cdot \mathrm{s}$ \\
\hline 42 & gAr Viscosity @ 2.4 bars (conv.) & $=B 41 * 100$ & 0.01261 & centipoise \\
\hline 43 & Max. Mass Flow to Rupture Disk & $=B 17^{*}(1-B 18)$ & 6404 & $\mathrm{lbm} / \mathrm{hr}$ \\
\hline 44 & Reynolds Number & $-6.31^{*} \mathrm{~B} 43 /\left(\mathrm{B} 37^{*} \mathrm{~B} 42\right)$ & 1550000 & \\
\hline 45 & $>$ Relative Roughness $(\theta / D)$ & 0.0009 & 0.0009 & \\
\hline 46 & Friction Factor Guess & $=0.25^{*}\left(\operatorname{LOG}\left(B 45 / 3.7+5.74 /\left(B 44^{\wedge} 0.9\right)\right)\right)^{\wedge}-2$ & 0.0194 & \\
\hline 47 & Friction Factor & $-0.25^{*}\left(\operatorname{LOG}\left(B 45 / 3.7+2.51 /\left(B 44^{*} B 46^{\wedge} 0.5\right)\right)\right)^{\wedge}-2$ & 0.0194 & \\
\hline 48 & Pressure Drop & $=0.00000336^{*} \mathrm{~B} 47^{*} \mathrm{~B} 38^{*}\left(\mathrm{~B} 43^{\wedge} 2\right) / \mathrm{B} 40 /\left(\mathrm{B} 37^{\wedge} 5\right)$ & 7.412 & psi \\
\hline 51 & & & & \\
\hline 52 & $\Delta \mathrm{P}$ Across Relief Valve Outlet & & & Units \\
\hline 53 & Inner Pipe Diameter (conv.) & $-B 54 / 12$ & 0.272 & $\mathrm{ft}$ \\
\hline 54 & $>$ Inner Pipe Diameter & 3.26 & 3.26 & in \\
\hline 55 & $>$ Equivalent Length & 51 & 51 & ft \\
\hline 56 & > gAr Density @ 2.0 bar \& Temp. & 6.292 & 6.292 & $\mathrm{mg} / \mathrm{cc}$ \\
\hline 57 & gAr Density @ 2.0 bar (conv.) & $-B 56 / 1000^{*} 62.428$ & 0.393 & $\mathrm{lb} \mathrm{m} / \mathrm{ft} \wedge 3$ \\
\hline 58 & > gAr Viscosity @ 2.0 bar \& Temp. & 0.000126 & 0.000126 & $\mathrm{~g} / \mathrm{cm}-\mathrm{s}$ \\
\hline 59 & gAr Viscosity @ 2.0 bar (conv.) & $=\mathrm{B} 58 * 100$ & 0.0126 & centipoise \\
\hline 60 & Max. Mass Flow to Relief Valve & $=\mathrm{B} 28$ & 5911 & $\mathrm{lbm} / \mathrm{hr}$ \\
\hline 61 & Reynolds Number & $-6.31^{*} \mathrm{~B} 60 /\left(\mathrm{B} 54^{*} \mathrm{~B} 59\right)$ & 908000 & \\
\hline 62 & $>$ Relative Roughness $(\theta / D)$ & 0.00055 & 0.00055 & \\
\hline 63 & Friction Factor Guess & $=0.25^{*}\left(\mathrm{LOG}\left(\mathrm{B} 62 / 3.7+5.74 /\left(\mathrm{B} 61^{\wedge} 0.9\right)\right)\right)^{\wedge}-2$ & 0.0177 & \\
\hline 64 & Friction Factor & $=0.25^{\star}\left(\operatorname{LOG}\left(B 62 / 3.7+2.51 /\left(B 61^{*} B 63^{\wedge} 0.5\right)\right)\right)^{\wedge}-2$ & 0.0176 & \\
\hline 65 & Pressure Drop & $=0.000000336^{*} \mathrm{~B} 64^{*} \mathrm{~B} 55^{*}\left(\mathrm{~B} 60^{\wedge} 2\right) / \mathrm{B} 57 /\left(\mathrm{B} 54^{\wedge} 5\right)$ & 0.728 & psi \\
\hline 66 & & & & \\
\hline
\end{tabular}




\section{Maximum Module Temperature Calculation 10/17/91}

\begin{tabular}{|c|c|c|c|c|}
\hline & A & B & $\mathbf{C}$ & $\mathbf{D}$ \\
\hline 67 & $\triangle \mathrm{P}$ Across Rupture Disk Outlet & & & Units \\
\hline 68 & Inner Pipe Diameter (conv.) & $-\mathrm{B} 69 / 12$ & 0.18 & $f t$ \\
\hline 69 & $>$ Inner Pipe Diameter & 2.157 & 2.157 & in \\
\hline 70 & $>$ Equivalent Length & 1.75 & 1.75 & $f t$ \\
\hline 71 & gAr Density @ 2.0 bar \& Temp. & $=\mathrm{B} 56$ & 6.292 & $\mathrm{mg} / \mathrm{cc}$ \\
\hline 72 & gAr Density @ 2.0 bar (conv.) & $=\mathrm{B} 71 / 1000^{*} 62.428$ & 0.393 & $\mathrm{lbm} / \mathrm{ft}^{\wedge} 3$ \\
\hline 73 & gAr Viscosity @ 2.0 bar \& Temp. & $=\mathrm{B} 58$ & 0.000126 & $\mathrm{~g} / \mathrm{cm}-\mathrm{s}$ \\
\hline 74 & gAr Viscosity @2.0 bar (conv.) & $=B 73 * 100$ & 0.0126 & centipoise \\
\hline 75 & Max. Mass Flow to Rupture Disk & $=\mathrm{B} 43$ & 6404 & $16 \mathrm{~m} / \mathrm{hr}$ \\
\hline 76 & Reynolds Number & $-6.31^{*} \mathrm{~B} 75 /\left(\mathrm{B} 69^{*} \mathrm{~B} 74\right)$ & 1490000 & \\
\hline 77 & > Relative Roughness (e/D) & 0.0009 & 0.0009 & \\
\hline 78 & Friction Factor Guess & $=0.25^{*}\left(\operatorname{LOG}\left(\mathrm{B} 77 / 3.7+5.74 /\left(\mathrm{B} 76^{\wedge} 0.9\right)\right)\right)^{\wedge}-2$ & 0.0194 & \\
\hline 79 & Friction Factor & $=0.25^{*}\left(\operatorname{LOG}\left(B 77 / 3.7+2.51 /\left(B 76^{*} B 78^{\wedge} 0.5\right)\right)\right)^{\wedge}-2$ & 0.0194 & \\
\hline 80 & Pressure Drop & $=0.00000336^{*} \mathrm{~B} 79^{*} \mathrm{~B} 70^{*}\left(\mathrm{~B} 75^{\wedge} 2\right) / \mathrm{B} 72 /\left(\mathrm{B} 69^{\wedge} 5\right)$ & 0.255 & psi \\
\hline \multicolumn{5}{|c|}{81 | 2} \\
\hline 82 & Change in Gas at Common Outlet to Outside & & & Units \\
\hline 83 & Pressure in Cryostat & $=\mathrm{B} 4$ & 19.75 & psig \\
\hline 84 & Pressure in Cryostat (conv.) & $=(B 83 / 14.696+1) * 1.01325$ & 2.375 & bars \\
\hline 85 & gAr Density @ 2.2 bars & $=\mathrm{B9}$ & 6.927 & $\mathrm{mg} / \mathrm{cc}$ \\
\hline 86 & gAr Density @ 2.4 bars & $=\mathrm{B} 10$ & 7.566 & $\mathrm{mg} / \mathrm{cc}$ \\
\hline 87 & gAr Density @ 2.375 \& Temp. & $=(\mathrm{B} 84-2.2) / 0.2^{*}(\mathrm{~B} 86-\mathrm{B} 85)+\mathrm{B} 85$ & 7.486 & $\mathrm{mg} / \mathrm{cc}$ \\
\hline 88 & Temp. at Common Outlet & $=\left(\mathrm{B} 2 * \mathrm{~B} 17+84^{*} \mathrm{~B} 189\right) /(\mathrm{B} 17+\mathrm{B} 189)$ & 139 & $\mathrm{~K}$ \\
\hline 89 & $>$ Pressure to Calculate Density & 1.5 & 1.5 & bars \\
\hline 90 & "> gAr Density@ @ 1.5 bars \& Now Temp. & 5.265 & 5.265 & $\mathrm{mg} / \mathrm{cc}$ \\
\hline 81 & "> gAr Viscosity @ 1.5 bars \& Now Temp. & 0.0001133 & 0.0001133 & $\mathrm{~g} / \mathrm{cm}-\mathrm{s}$ \\
\hline 92 & gAr Viscosity @ 1.5 bar (conv.) & $=B 91 * 100$ & 0.01133 & centipoise \\
\hline 93 & & & & \\
\hline
\end{tabular}




\section{Maximum Module Temperature Calculation}

$10 / 17 / 91$

\begin{tabular}{|c|c|c|c|c|}
\hline & A & B & C & D \\
\hline 94 & $\Delta P$ Across Relief Valve & & & Units \\
\hline 95 & $>$ Critical Ratio (Pcr/P1) for Argon & 0.487 & 0.487 & \\
\hline 96 & $>$ Specific Heat Ratio (k) for Argon & $=\mathrm{B} 168$ & 1.69 & \\
\hline 97 & $>$ Area of $2^{n} \times 3^{n}$ Relief Valve & 2.29 & 2.29 & $\operatorname{in}^{\wedge} 2$ \\
\hline 98 & Flow Through Relief Valve & $=\mathrm{B} 28$ & 5911 & $\mathrm{lbm} / \mathrm{hr}$ \\
\hline 99 & Flowing Temperature & $=1.8^{*} \mathrm{~B} 2$ & 279 & $\operatorname{dog} R$ \\
\hline 100 & $>$ Compressibility Factor & 1 & 1 & \\
\hline 101 & $>$ Nozzle Coefficient for type 93T & 0.939 & 0.939 & \\
\hline 102 & Flowing Inlet Pressure (P1) & $=\mathrm{B} 4+14.696-\mathrm{B} 33$ & 32.55 & psia \\
\hline 103 & $>$ Molecular Weight of Argon & 39.948 & 39.95 & $\mathrm{~g} / \mathrm{mol}$ \\
\hline 104 & Critical Pressure (Pcr) & $=\mathrm{B} 95^{\circ} \mathrm{B} 102$ & 15.85 & psia \\
\hline 105 & Outlet Pressure (P2) (using delta p's) & $-14.696+\mathrm{B} 154+\mathrm{B} 133+\mathrm{B} 65$ & 26.05 & psia \\
\hline 106 & Pressure Ratio $\left(\mathrm{P}^{*} / \mathrm{P} 1\right)$ & $=\left(B 102-0.55^{*}\left((B 102-B 105)^{\wedge} 0.98\right)\right) / B 102$ & 0.894 & \\
\hline 107 & Theoretical Factor (F*) (using P2) & $=\operatorname{SQRT}\left((\mathrm{B} 96 /(\mathrm{B} 96-1))^{*}\left(\mathrm{~B} 106^{\wedge}(2 / \mathrm{B} 96)-\mathrm{B} 106^{\wedge}((\mathrm{B} 96+1) / \mathrm{B} 96)\right)\right)$ & 0.309 & \\
\hline 108 & Max. Theoretical Relief Flow (using $F^{*}$ ) & $=735^{*} \mathrm{~B} 97^{*} \mathrm{~B} 101^{*} \mathrm{~B} 102^{*} \mathrm{~B} 107^{*} \mathrm{SQRT}(\mathrm{B} 103 / \mathrm{B} 99 / \mathrm{B} 100)$ & 6021 & $\mathrm{lbm} / \mathrm{hr}$ \\
\hline 109 & Theoretical Percent of Relief Flow & $=\mathrm{B} 108 / \mathrm{B} 17$ & 0.4889 & \\
\hline 110 & Pressure Drop Across Relief Valve & $=\mathrm{B} 102-\mathrm{B} 105$ & 6.494 & psi \\
\hline 111 & & & & \\
\hline
\end{tabular}


Maximum Module Temperature Calculation 10/17/91

\begin{tabular}{|c|c|c|c|c|}
\hline & A & B & c & D \\
\hline 112 & $\Delta \mathrm{P}$ Across Common Outlet to Platform Bayonet & & & Units \\
\hline 113 & $>$ Inner Pipe Diameter & 0.355 & 0.355 & $\mathrm{ft}$ \\
\hline 114 & Inner Pipe Diameter (conv.) & $=\mathrm{B} 113^{*} 12$ & 4.26 & in \\
\hline 115 & $>$ Equivalent Length & 273 & 273 & $f t$ \\
\hline 116 & gAr Density @ 1.5 bar \& New Temp. & $=\mathrm{B} 90$ & 5.265 & $\mathrm{mg} / \mathrm{cc}$ \\
\hline 117 & gAr Density @ 1.5 bar (conv.) & $=B 116 / 1000^{\circ} 62.428$ & 0.329 & $\mathrm{bm} / \mathrm{ft}^{\wedge} 3$ \\
\hline 118 & $>$ gN2 Gas Density @ 1.5 bar \& New Temp. & 3.72712 & 3.727 & $\mathrm{mg} / \mathrm{cc}$ \\
\hline 119 & gN2 Gas Density @ 1.5 bar (conv.) & $-\mathrm{B} 118 / 1000^{\circ} 62.428$ & 0.233 & $\mathrm{lb} \mathrm{m} / \mathrm{ft}^{\wedge} 3$ \\
\hline 120 & Gas Mixture Density @ 1.5 bar & $=\left(B 17^{*} \mathrm{~B} 117+\mathrm{B} 127^{*} \mathrm{~B} 119\right) / \mathrm{B} 128$ & 0.308 & $\mathrm{Ib} \mathrm{m} / \mathrm{ft}^{\wedge} 3$ \\
\hline 121 & gArViscosity @ 1.5 bar \& Now Tomp. & B91 & 0.0001133 & $\mathrm{~g} / \mathrm{cm}-\mathrm{s}$ \\
\hline 122 & gAr Viscosity @ 1.5 bar (conv.) & $-\mathrm{B} 121 * 100$ & 0.01133 & centipoise \\
\hline 123 & $>$ gN2 Viscosity @ 1.5 bar \& New Temp. & 0.000093482 & 0.00009348 & $\mathrm{~g} / \mathrm{cm}-\mathrm{s}$ \\
\hline 124 & gN2 Viscosity @ 1.5 bar (conv.) & $-\mathrm{B} 123^{\circ} \cdot 100$ & 0.009348 & centipoise \\
\hline 125 & Mixture Viscosity @1.5 bar & $-\left(\mathrm{B} 17^{*} \mathrm{~B} 122+\mathrm{B} 127^{*} \mathrm{~B} 124\right) / \mathrm{B} 128$ & 0.01089 & centipoise \\
\hline 126 & Max. Mass Flow of Argon Gas & $-\mathrm{B} 17$ & 12315 & $\mathrm{lbm} / \mathrm{hr}$ \\
\hline 127 & Max. Flow of Nitrogen Gas & $=\mathrm{B} 189$ & 3477 & $\mathrm{lbm} / \mathrm{hr}$ \\
\hline 128 & Mass Flow of Mixture & $-B 126+B 127$ & 15793 & $\mathrm{lbm} / \mathrm{hr}$ \\
\hline 129 & Reynolds Number & $=6.31^{*} \mathrm{~B} 128 /\left(\mathrm{B} 114^{*} \mathrm{~B} 125\right)$ & 2150000 & \\
\hline 130 & $>$ Relative Roughness $(\theta / D)$ & 0.0004 & 0.0004 & \\
\hline 131 & Friction Factor Guess & $=0.25^{*}\left(\operatorname{LOG}\left(B 130 / 3.7+5.74 /\left(B 129^{\wedge} 0.9\right)\right)\right)^{\wedge}-2$ & 0.0163 & \\
\hline 132 & Friction Factor & $=0.25^{\circ}\left(\operatorname{LOG}\left(\mathrm{B} 130 / 3.7+2.51 /\left(\mathrm{B} 129^{*} \mathrm{~B} 131^{\wedge} 0.5\right)\right)\right)^{\wedge}-2$ & 0.0162 & \\
\hline 133 & Pressure Drop & $=0.00000336^{*} \mathrm{~B} 132^{*} \mathrm{~B} 115^{*}\left(\mathrm{~B} 128^{\wedge} 2\right) / \mathrm{B} 120 /\left(\mathrm{B} 114^{\wedge} 5\right)$ & 8.579 & psi \\
\hline 134 & & & & \\
\hline
\end{tabular}




\begin{tabular}{|c|c|c|c|c|}
\hline & A & B & $\mathbf{C}$ & D \\
\hline 135 & $\Delta \mathrm{P}$ from Platform Bayonet to Outside & & & Units \\
\hline 136 & $>$ Inner Pipe Diameter & 0.53 & 0.53 & $\mathrm{ft}$ \\
\hline 137 & Inner Pipe Diameter (conv.) & $=\mathrm{B} 136^{* 12}$ & 6.36 & in \\
\hline 138 & $>$ Equivalent Length & 516 & 516 & $f t$ \\
\hline 139 & gAr Gas Density @ 1.5 bar \& New Temp. & $=\mathrm{B} 90$ & 5.265 & $\mathrm{mg} / \mathrm{cc}$ \\
\hline 140 & gAr Gas Density @ 1.5 bar (conv.) & $=\mathrm{B} 139 / 1000 * 62.428$ & 0.329 & $\mathrm{bm} / \mathrm{ft}^{\wedge} 3$ \\
\hline 141 & gN2 Gas Density @ 1.5 bar \& New Temp. & $=\mathrm{B} 118$ & 3.727 & $\mathrm{mg} / \mathrm{cc}$ \\
\hline 142 & gN2 Gas Density @ 1.5 bar (conv.) & $=\mathrm{B} 141 / 1000 * 62.428$ & 0.233 & $\mathrm{lbm} / \mathrm{ft}^{\wedge} 3$ \\
\hline 143 & Gas Mixture Density @1.5 bar & $=\left(\mathrm{B} 17^{*} \mathrm{~B} 140+\mathrm{B} 189^{*} \mathrm{~B} 142\right) / \mathrm{B} 149$ & 0.308 & $\mathrm{lbm} / \mathrm{ft}^{\wedge} 3$ \\
\hline 144 & gAr Viscosity @ 1.5 bar \& New Temp. & $=\mathrm{B} 121$ & 0.0001133 & $\mathrm{~g} / \mathrm{cm}-\mathrm{s}$ \\
\hline 145 & gAr Viscosity @ 1.5 bar (conv.) & $=\mathrm{B} 144^{*} 100$ & 0.01133 & centipoise \\
\hline 146 & gN2 Viscosity@1.5 bar \& Now Tomp. & $=\mathrm{B} 123$ & 0.00009348 & $\mathrm{~g} / \mathrm{cm}-\mathrm{s}$ \\
\hline 147 & gN2 Viscosity @ 1.5 bar (conv.) & $=\mathrm{B} 146 * 100$ & 0.009348 & centipoise \\
\hline 148 & Gas Mixture Viscosity @1.5 bar & $=\left(\mathrm{B} 17^{*} \mathrm{~B} 145+\mathrm{B} 189^{*} \mathrm{~B} 147\right) / \mathrm{B} 149$ & 0.01089 & centipoise \\
\hline 149 & Max. Mass Flow of Gas Mixture & $=\mathrm{B} 128$ & 15793 & $\mathrm{lbm} / \mathrm{hr}$ \\
\hline 150 & Reynolds Number & $=6.31 * \mathrm{~B} 149 /\left(\mathrm{B} 137^{*} \mathrm{~B} 148\right)$ & 1440000 & \\
\hline 151 & $>$ Relative Roughness (e/D) & 0.00027 & 0.00027 & \\
\hline 152 & Friction Factor Guess & $=0.25^{\star}\left(\operatorname{LOG}\left(\mathrm{B} 151 / 3.7+5.74 /\left(\mathrm{B} 150^{\wedge} 0.9\right)\right)\right)^{\wedge}-2$ & 0.0153 & \\
\hline 153 & Friction Factor & $=0.25^{*}\left(\mathrm{LOG}\left(\mathrm{B} 151 / 3.7+2.51 /\left(\mathrm{B} 150^{*} \mathrm{~B} 152^{\wedge} 0.5\right)\right)\right)^{\wedge}-2$ & 0.0152 & \\
\hline 154 & Pressure Drop & $=0.00000336^{*} \mathrm{~B} 153^{*} \mathrm{~B} 138^{*}\left(\mathrm{~B} 149^{\wedge} 2\right) / \mathrm{B} 143 /\left(\mathrm{B} 137^{\wedge} 5\right)$ & 2.049 & psi \\
\hline 155 & & & & \\
\hline 156 & Summation of Equivalent $\Delta \mathrm{PS}$ & & & Units \\
\hline 157 & Relief Valve Inlet Pressure Drop & $=\mathrm{B} 33$ & 1.9 & psi \\
\hline 158 & Relief Valve Outlet Pressure Drop & $=\mathrm{B} 65$ & 0.728 & psi \\
\hline 159 & Relief Valve Pressure Drop & $=\mathrm{B} 110$ & 6.494 & psi \\
\hline 160 & Relief Valve/Disk Branch & $=\mathrm{B} 33+\mathrm{B} 65+\mathrm{B} 110$ & 9.122 & psi \\
\hline 161 & Rupture Disk Inlet Pressure Drop & $=\mathrm{B} 48$ & 7.412 & psi \\
\hline 162 & Rupture Disk Outlet Pressure Drop & $=\mathrm{B} 80$ & 0.255 & psi \\
\hline 163 & Rupture Disk Pressure Drop & $=\mathrm{B} 157+\mathrm{B} 158+\mathrm{B} 159-\mathrm{B} 161-\mathrm{B} 162$ & 1.455 & psi \\
\hline 164 & Common Outlet Pressure Drop & $=\mathrm{B} 133$ & 8.579 & psi \\
\hline 165 & Platform to Outside Pressure Drop & $=\mathrm{B} 154$ & 2.049 & psi \\
\hline 166 & & & & \\
\hline
\end{tabular}




\begin{tabular}{|c|c|c|c|c|}
\hline & A & B & C & D \\
\hline 167 & $\triangle P$ Across Rupture Disk & & & Units \\
\hline 168 & >Argon Specific Heat Ratio (k) & 1.691 & 1.691 & \\
\hline 169 & Critical Ratio & $=(2 /(\mathrm{B} 168+1)))^{\wedge}(\mathrm{B} 168 /(\mathrm{B} 168-1))$ & 0.484 & \\
\hline 170 & $>$ Area of $3^{n}$ Rupture Disk & $=3.14159^{*}\left(3^{\wedge} 2\right) / 4$ & 7.069 & $\operatorname{in}^{\wedge} 2$ \\
\hline 171 & Flow Through Rupture Disk & $=\mathrm{B} 43$ & 6404 & $1 \mathrm{bm} / \mathrm{hr}$ \\
\hline 172 & Flowing Temperature & $=1.8 * \mathrm{~B} 2$ & 279 & $\operatorname{deg} R$ \\
\hline 173 & $>$ ASME Coofficient $(K)$ & 0.62 & 0.62 & \\
\hline 174 & Pressure Ratio (Pe/Po) & $=\mathrm{B} 179 / \mathrm{B} 176$ & 0.946 & \\
\hline 175 & gAr Flow Constant for Subsonic Flow(C1) & $=\operatorname{SQRT}\left(2^{*} 32.2 / 1545^{*}(\mathrm{~B} 168 /(\mathrm{B} 168-1))^{*}\left(\mathrm{~B} 174^{\wedge}(2 / \mathrm{B} 168)-\mathrm{B} 174^{\wedge}\right.\right.$ & $0 . \overline{046}$ & \\
\hline 176 & Flowing Inlet Pressure (Po) & $=\mathrm{B} 4+14.696-\mathrm{B} 48$ & 27.03 & psia \\
\hline 177 & $>$ Molecular Weight of Argon & 39.948 & 39.948 & $\mathrm{~g} / \mathrm{mol}$ \\
\hline 178 & Critical Pressure (Pcr) & $=\mathrm{B} 169 * \mathrm{~B} 176$ & 13.08 & psia \\
\hline 179 & Outlet Pressure $\left(\mathrm{P}_{\theta}\right)$ (using delta $\mathrm{p}^{\prime} \mathrm{s}$ ) & $=14.696+\mathrm{B} 154+\mathrm{B} 133+\mathrm{B} 80$ & 25.58 & psia \\
\hline 180 & Pressure Drop Across Rupture Disk & $=\mathrm{B} 176-\mathrm{B} 179$ & $1.45 \overline{5}$ & psi \\
\hline 181 & Maximum Theoretical Rupture Disk Flow & $=\mathrm{B} 170^{*} \mathrm{~B} 173^{*} \mathrm{~B} 175^{*} \mathrm{~B} 176^{*} \mathrm{SQRT}(\mathrm{B} 177 / \mathrm{B} 172)^{*} 60^{*} 60$ & 7458 & $\mathrm{lbm} / \mathrm{hr}$ \\
\hline 182 & Actual Rupture Disk Flow & $=\mathrm{B} 43$ & 6404 & $\mathrm{lbm} / \mathrm{hr}$ \\
\hline \multicolumn{5}{|c|}{\begin{tabular}{l|l|}
83 & \\
18 & \\
\end{tabular}} \\
\hline 184 & Maximum Flow from Condensers & & & units \\
\hline 185 & $>$ Max. Flow of Liquid Nitrogen & 9.3 & 9.3 & gpm \\
\hline 186 & Max. Flow of Liquid Nitrogen (conv.) & $=\mathrm{B} 185 / 7.48$ & 1.24 & $\mathrm{ft} \wedge 3 / \mathrm{min}$ \\
\hline 187 & Density of LN2@ 3.5 atm & 0.747 & 0.747 & $g / c c$ \\
\hline 188 & Density of LN2 (conv.) & $=\mathrm{B} 187^{*} 62.4$ & 46.6128 & $16 \mathrm{~m} / f t^{\wedge} 3$ \\
\hline 189 & Mass Flow of LN2 & $=\mathrm{B} 186 * \mathrm{~B} 188 * 60$ & 3477 & $\mathrm{lbm} / \mathrm{hr}$ \\
\hline \multicolumn{5}{|c|}{190} \\
\hline 191 & Notes: & & & \\
\hline \multicolumn{5}{|c|}{192 " $>$ indicates that this value must be changed for a } \\
\hline 193 & new temperature & & & \\
\hline \multicolumn{5}{|c|}{194} \\
\hline \multicolumn{5}{|c|}{195 > indicates variable not requiring change for new } \\
\hline \multirow{2}{*}{\multicolumn{5}{|c|}{\begin{tabular}{|l|l|}
196 & temperature \\
197 & \\
\end{tabular}}} \\
\hline & & & & \\
\hline \multicolumn{5}{|c|}{ indicates the previous value converted to new } \\
\hline 199 & units & & & \\
\hline
\end{tabular}




\section{Calculation of Max. Flowrate from $\mathrm{CC}$ to $\mathrm{ECN}$}

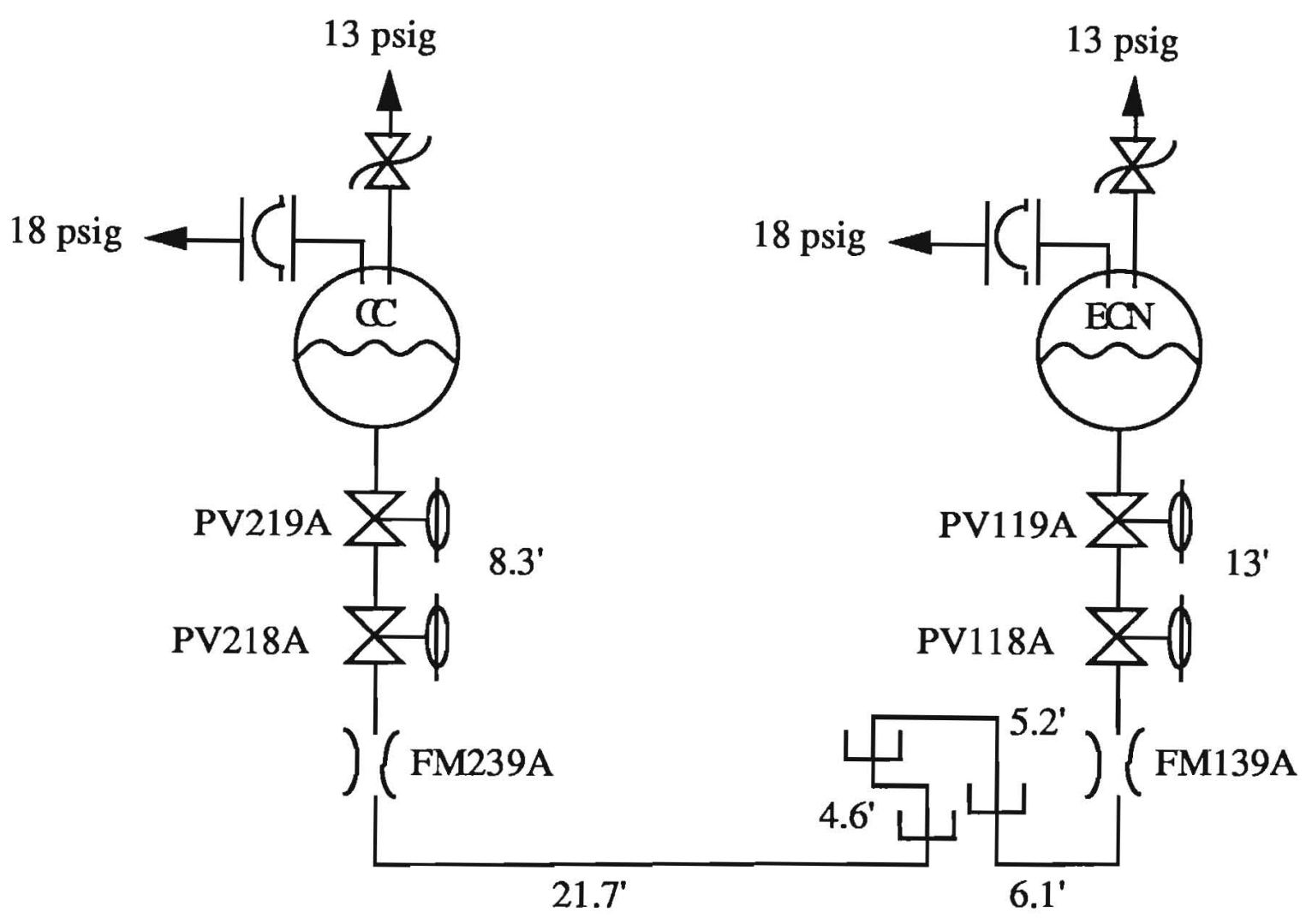

$\mathrm{d}=1.682$ in $=0.1402 \mathrm{ft}$

$A=0.01543 \mathrm{ft}^{2}$
[1 1/2" SCH. 10 inner pipe dia.] [cross-sectional area] 


\begin{tabular}{|l|c|c|c|}
\hline $\begin{array}{l}\text { Reference: drawings } \\
\text { from Tony Parker }\end{array}$ & CC drain line & ECN drain line & TOTAL \\
\hline \# of elbows, $90^{\circ}$ & 8 & 19 & 27 \\
\hline \# of elbows, $45^{\circ}$ & 1 & 1 & 2 \\
\hline \# of tees, branch & 1 & 0 & 1 \\
\hline \# of tees, thru & 1 & 0 & 1 \\
\hline
\end{tabular}




\section{Calculation of Equivalent Length}

Calculate the equivalent length of the piping from the inner vessel of the $\mathrm{CC}$ to the inner vessel of the $\mathrm{ECN}$.

$\mathrm{L}_{\text {piping }}=\mathrm{L}_{1.7}$ " dia. $+\mathrm{L}_{1.0}$ " dia.

$\mathrm{L}_{1.7}$ " dia. $=6.8 \mathrm{ft}+8.3 \mathrm{ft}(\mathrm{CC}$ drain line $)+21.7 \mathrm{ft}(\mathrm{CC}$ to rotary bayonet assembly) $+4.6 \mathrm{ft}+5.2 \mathrm{ft}$ (rotary U-tube dimensions) $+6.1 \mathrm{ft}+13 \mathrm{ft}(\mathrm{ECN}$ drain line $)=65.7 \mathrm{ft}$ total.

Equivalent lengths of the two flowmeters are accounted for by including two 4 foot lengths of 1.0 " diameter piping. Convert 1.0 " diameter equivalent length to 1.7 " dia. equivalent length:

Reference: Crane Technical Paper No. 410

(1" SCH. 40 to $11 / 2$ " SCH. 10)

$\mathrm{L}_{1.0 \text { dia. }}=\left(\frac{1.682}{1.049}\right)^{5} \times 4^{\prime}=42.4^{\prime}$

$\mathrm{L}_{\text {piping }}=65.7 \mathrm{ft}+2(42.4 \mathrm{ft})=150.5 \mathrm{ft}$ total

Convert elbows and tees into equivalent lengths of pipe.

$\mathrm{L}_{\text {fittings }}=[27(20)+2(14)+1(60)+1(20)] \times 0.1402 \mathrm{ft}=90.8 \mathrm{ft}$

$\mathrm{L}_{\mathrm{eq}}=150.5 \mathrm{ft}+90.8 \mathrm{ft}=241.3 \mathrm{ft}$ 


\section{Calculation of Resistance Coefficient}

Reference: Crane Technical Paper No. 410

Calculate the resistance coefficient for the piping and fittings.

$K_{\text {piping, fittings }}=f\left(\frac{L_{e q}}{d}\right)$

let $\mathrm{f}=0.02136 \quad$ [friction factor guess]

$\mathrm{K}_{\text {piping, fittings }}=0.02136\left(\frac{241.3 \mathrm{ft}}{0.1402 \mathrm{ft}}\right)=36.76$

Include inlet and outlet losses (ref. Kelly Dixon).

$\mathrm{K}_{\text {inlet }}=0.5$

$\mathrm{K}_{\text {outlet }}=1.0$

Calculate resistance coefficient for the valves, where $C_{v}$, the valve coefficient, is 34 for PV218A (CC), and 41 for PV118A (ECN).

$\mathrm{K}_{\text {valves }}=\left(\frac{29.9 \mathrm{~d}^{2}}{\mathrm{C}_{\mathrm{v}}}\right)^{2}=\left(\frac{29.9(1.682)^{2}}{34}\right)^{2}+\left(\frac{29.9(1.682)^{2}}{41}\right)^{2}=10.45$ where the diameter, $d$, is in inches, not feet.

$\sum \mathrm{K}=36.76+1.5+10.45=\mathbf{4 8 . 7 1}$ 


\section{Driving Pressure}

Calculate the differential pressure available under relieving conditions. max. head available $=17 \mathrm{ft}(\mathrm{CC}$ completely full $)$

Calculate the pressure due to the head.

Density of liquid argon @ 19.75 psig = $1.337 \mathrm{~g} / \mathrm{cc}$, which corresponds to a specific weight of $0.580 \mathrm{psi} / \mathrm{ft}$.

$\Delta \mathrm{p}$ due to head $=17 \mathrm{ft} \times 0.580 \mathrm{psi} / \mathrm{ft}=9.86 \mathrm{psi}$

$\Delta \mathrm{p}_{\text {relieving }}=(\mathrm{CC}$ pressure $)-(\mathrm{ECN}$ pressure $)+($ head pressure $)$

$$
=27.7 \mathrm{psia}-34.45 \mathrm{psia}+9.86 \mathrm{psi}=3.11 \mathrm{psid}
$$

$\Delta \mathrm{p}_{\text {available }}=\Delta \mathrm{p}_{\text {relieving }}=3.11 \mathrm{psid}$ 


\section{Calculation of Flowrate}

Calculate the flowrate, q, by rearranging Darcy's formula (ref. Crane 410). modified Darcy's formula:

$\Delta \mathrm{p}=\frac{\rho \Sigma \mathrm{K}}{144} \frac{\mathrm{v}^{2}}{2 \mathrm{~g}_{\mathrm{c}}}$

where: $\Delta \mathrm{p}$ is in psid,

$\rho$ is in $1 b_{m} / \mathrm{ft}^{3}$,

and $\mathrm{v}$ is in $\mathrm{ft} / \mathrm{s}$.

(144 is a conversion factor of in $^{2} / \mathrm{ft}^{2}$.)

Rearrange to solve for the velocity, $v$.

$v=\sqrt{\frac{2 \mathrm{~g}_{\mathrm{c}}(144 \Delta \mathrm{p})}{\rho \Sigma \mathrm{K}}}=\sqrt{\frac{2\left(32.174 \frac{1 \mathrm{~b}_{\mathrm{m}}-\mathrm{ft}}{1 \mathrm{~b}_{\mathrm{f}} \mathrm{s}^{2}}\right)\left(144 \frac{\mathrm{in}^{2}}{\mathrm{ft}^{2}}\right) \Delta \mathrm{p}_{\text {available }}}{\left(83.47 \frac{1 \mathrm{~b}_{\mathrm{m}}}{\mathrm{ft}^{3}}\right)(48.71)}}$

$\mathrm{v}=1.510 \sqrt{\Delta \mathrm{p}_{\text {available }}}$

where $\mathrm{v}$ is in $\mathrm{ft} / \mathrm{s}$ if $\Delta \mathrm{p}_{\text {available }}$ is in psid.

$\mathrm{v}=1.510 \sqrt{\Delta \mathrm{p}_{\mathrm{available}}}=1.510 \sqrt{3.11}=2.663 \frac{\mathrm{ft}}{\mathrm{s}}$ 
Solve for $\mathrm{q}$, the flow rate in cfs:

$q=A v=\left(0.01543 \mathrm{ft}^{2}\right)\left(2.663 \frac{\mathrm{ft}}{\mathrm{s}}\right)=0.0411 \mathrm{cfs}$

Convert q to gpm:

$\mathrm{q}=0.0411 \frac{\mathrm{ft}^{3}}{\mathrm{sec}} \times \frac{\mathrm{gal}}{0.13368 \mathrm{ft}^{3}} \times \frac{60 \mathrm{sec}}{\mathrm{min}}$

$\mathrm{q}=18.45 \mathrm{gpm}$

\section{Check Friction Factor}

$\operatorname{Re}_{\mathrm{d}_{\text {relieving }}}=\frac{\rho \mathrm{V}_{\mathrm{rel} .} \mathrm{d}}{\mu}$

$\mu=2.4185 \times 10^{-3} \frac{\mathrm{g}}{\mathrm{cm}-\mathrm{s}} @$ sat. $1.3 \mathrm{bars}=1.6252 \times 10^{-4} \frac{1 \mathrm{~b}_{\mathrm{m}}}{\mathrm{ft}-\mathrm{s}}$

$\mathrm{q}_{\text {relieving }}=18.45 \mathrm{gpm}$

$\mathrm{v}_{\text {relieving }}=2.663 \mathrm{ft} / \mathrm{s}$ (from before)

$\operatorname{Re}_{\mathrm{d}_{\text {relieving }}}=\frac{\left(83.47 \frac{\mathrm{lb}}{\mathrm{ft}_{\mathrm{m}}^{3}}\right)\left(2.663 \frac{\mathrm{ft}}{\mathrm{s}}\right)(0.1402 \mathrm{ft})}{1.6252 \times 10^{-4} \frac{1 \mathrm{~b}_{\mathrm{m}}}{\mathrm{ft}-\mathrm{s}}}=1.918 \times 10^{5}$

Assuming a value of 0.00015 roughness for commercial steel pipe, the relative roughness is 0.001 , and the friction factor is 0.02136 , which agrees with the assumption. 


\section{TK Solver Plus Analysis}

The following two pages are printouts of the variable and rule sheet from a TK Solver model set up to verify the hand calculations for the maximum flow rate to the ECN from the CC. The first page is the variable sheet, which shows the typed inputs and calculated outputs for various parameters. The complete solution requires guessing a number for the friction factor. The program then iterates to find the exact solution. Also note that the columns have a set width, so that not all of the entries are shown completely, specifically, $g$ has units of $1 \mathrm{bm}-\mathrm{ft} / \mathrm{lbf}-\mathrm{s}^{\wedge} 2$. The second sheet is the rule sheet, which shows the various formulas used. The complete model has been saved in the Co-op Mac, under the name "TK CC Flow to ECN". 


\begin{tabular}{|c|c|c|c|c|}
\hline \multirow{4}{*}{ St Input } & Name & output & Unit & comment \\
\hline & $\mathrm{D}$ & & in & inner plpe dia. \\
\hline & d & .14016667 & ft & inner pipe dia. (converted) \\
\hline & A & .01543046 & $\mathrm{ft}^{\wedge} 2$ & cross-sectional area \\
\hline 27 & Els90 & & & number of $90^{\circ}$ elbows \\
\hline 2 & E1s45 & & & number of $45^{\circ}$ elbows \\
\hline 1 & TeesBr & & & number of tees, branch \\
\hline 1 & Teesth & & & number of tees, thru \\
\hline 150.5 & Lpiping & & ft & equivalent length of 1.7 " piping \\
\hline & Lfittin & 90.828 & ft & equivalent length of fittings \\
\hline & Leq & 241.328 & ft & equivalent length \\
\hline & $\mathrm{f}$ & .02135622 & & friction factor (guess) \\
\hline .5 & Kinlet & & & inlet resistance \\
\hline 1 & Koutlet & & & outlet resistance \\
\hline & Kpiping & 36.769466 & & resistance of piping \\
\hline 34 & Cvofcc & & & coeff. of CC valve \\
\hline 41 & CvofECN & & & coeff. of ECN valve \\
\hline & Kvalves & 10.446724 & & resistance of valves \\
\hline & Sumk & 48.71619 & & summation of resistances \\
\hline 17 & elev & & ft & max. head available \\
\hline 83.47 & rho & & $1 \mathrm{bm} / \mathrm{ft} \wedge 3$ & density of argon \\
\hline & Spwt & .57965278 & $\mathrm{psi} / \mathrm{ft}$ & specific weight of argon \\
\hline & DPhead & 9.8540972 & psi & delta $p$ from head \\
\hline & DPrel & 3.1040972 & psi & delta $\mathrm{p}$ relieving \\
\hline & DPavail & 3.1040972 & psi & delta $\mathrm{p}$ available \\
\hline & Vrel & 2.6595892 & $\mathrm{ft} / \mathrm{s}$ & relieving velocity \\
\hline & $\mathrm{Re}$ & 191462.14 & & Reynold's number \\
\hline .00016252 & visc & & $1 \mathrm{bm} / \mathrm{ft}-\mathrm{s}$ & viscosity \\
\hline .00015 & e & & & roughness coefficient \\
\hline & rough & .00107015 & & relative roughness (e/d) \\
\hline & pi & 3.14159 & & pi constant \\
\hline & 9 & 32.174 & $1 \mathrm{bm}-\mathrm{ft} / 1 \mathrm{~b}$ & gc conversion constant \\
\hline & q & 18.419523 & gpm & flow rate \\
\hline
\end{tabular}


S Rule

* $\mathrm{pi}=3.14159 \quad$ "constant for $\mathrm{pi}$

$\star \mathrm{g}=32.174$

"constant for gc, in units of (lbm-ft)/(lbf-s^2)

$\star \mathrm{d}=\mathrm{D} / 12$ "conversion of inner pipe dia. from inches to feet

$\star \mathrm{A}=\mathrm{pi}{ }^{\star}\left(\mathrm{d}^{\wedge} 2\right) / 4 \quad$ "cross-sectional area of pipe Lfittings $=\mathrm{d}^{\star}(\mathrm{Els} 90 \star 20+\mathrm{Els} 45 \star 14+\mathrm{TeesBr} \star 60+\mathrm{TeesTh} \star 20) \quad$ "equivalent length

* Leq $=$ Lpiping + Lfittings "total equivalent length of $1.7 "$ dia piping

* Kpiping $=f \star L e q / d \quad$ "resistance coeff. for piping and fittings

$\star$ Kvalves $=\left(29.9 *\left(D^{\wedge} 2\right) / \text { CvofCC }\right)^{\wedge} 2+\left(29.9 *\left(D^{\wedge} 2\right) / C v o f E C N\right) \wedge 2 \quad$ "resistance of

$\star$ SumK $=$ Kpiping+Kvalves+Kinlet +Koutlet "summation of $K$ coeff.

* Spwt $=$ rho/144 "specific weight at correct density

* DPhead $=$ elev * Spwt "delta $\mathrm{p}$ due to max. head pressure

* DPrel $=-19.75+13+$ DPhead "delta $p$ due to elevation difference

* DPavail = DPrel "available differential driving pressure

$\star$ DPavail $=\left(\right.$ rho* $\left.\mathrm{SumK}^{\star}\left(\operatorname{Vrel}^{\wedge} 2\right)\right) /\left(144^{\star} 2^{\star} \mathrm{g}\right) \quad$ "modified Darcy's formula

* Vrel $=\left(\mathrm{q}^{\star} .13368 / 60\right) / \mathrm{A} \quad$ "velocity in $\mathrm{ft} / \mathrm{s}$ from flow rate in $\mathrm{gpm}$

* $\mathrm{Re}=\mathrm{rho}$ *Vrel*d/visc "Reynold's number for pipe flow

* rough $=\mathrm{e} / \mathrm{d} \quad$ "relative roughness for commercial steel of $11 / 2$ " dia.

$\star 1 /\left(f^{\wedge} .5\right)=-2.0 * \log \left((\right.$ rough $\left./ 3.7)+\left(2.51 /\left(\operatorname{Re}^{\star}\left(f^{\wedge} .5\right)\right)\right)\right) \quad$ "Moody chart 


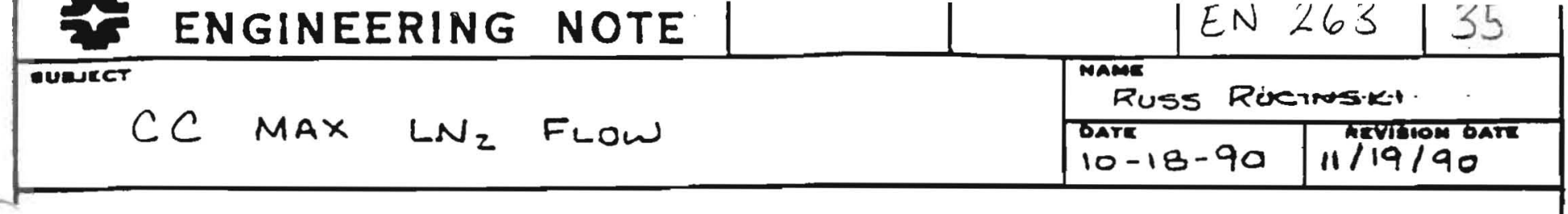

From COMPARING equivalent lengths, it IS CLEAR THAT ALMOST ALL PRESSURE DROP WILL OCCUR ACROSS

PV-201N. PV-201-N WITH $C_{V}=1.25$ WILL LIMIT LN 2 FLoW.

* * The same argument will apply to PVIOIN on the ECN.

KN 14 Aug 91

DRIVING PRESSURE

DÜRING COOLOOWWN THE LN 2 DEWAR PRESSURE IS. TQ PSIA [REFERENCE CC Cryostat ProceDURes Par. 7.4.1]

head DUE..TO ELEvation

CE Elevation LN $L$ ET WAR $\Rightarrow$ 749'-O"

DA. OF $L N_{2}$ DEWAR $=8.3 \mathrm{Ft}$

elevation top of LN 2 in Dewar is $753^{\prime}-2^{\prime \prime}$

elevation of CL condensing coils is $\frac{731: 2 \text { " }}{22 \mathrm{Ft} .}$

Convert it this head to PSI

$$
\begin{aligned}
& 22 \mathrm{Ft} \times \rho_{L N_{2}}(\text { PeCS }=44 \mathrm{FSLA})=22 \mathrm{Ft} \cdot\left(47.2 \frac{\mathrm{lbm}}{\mathrm{Ft}^{3}}\right)\left(\frac{1 \mathrm{Ft}^{2}}{144 \mathrm{FN}^{2}}\right) . \\
& =7.2 \mathrm{PSI}
\end{aligned}
$$

$$
\begin{aligned}
& \text { DRIVING PRESSURE }=\left(L N_{2} \text { DEWAR PRESS }\right)+(\text { HEAD })-[\text { CCMmMP }+3 \text { PSI }] \\
& =(70 \text { PSIA })+(7.2 \text { PSI })-[30 \text { PSIA }+3 \text { PI I }] \\
& \Delta P_{\text {max }}=44.2 \mathrm{PSI}
\end{aligned}
$$


ENGINEERING NOIL

BUDgET

$$
\text { CC. MAX. LN } \mathrm{N}_{2} \text { FLOW. }
$$

Ane

Russ Rucinski

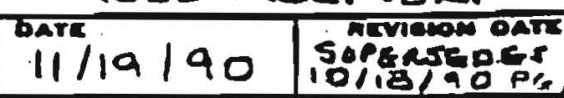

calculate maximum flow

Assumptions:

1) CondenSER Completely SEVERS

2) Neglect heat leak in transfer lune

3) PRESSURE. DROP THRU LINE IS NEGLIGIBLE (CONSGUative) le, PV-2O1-N LIMITS FlOW

4) Saturated $L N_{2}$

REFERENCE: FLOW EQUATIONS FOR SIZING CONTROL VALES is A standard ISA-575.01-1985

SECTION 5 APPLIES, INCOMPRESSIBLE FLUIO-CHOKED FLOW OF VAPORIZING LIQUID (FLASHING ACROSS PV-ZOI.N)

$$
q_{\text {max }}=N_{1} F_{L} C_{V} \sqrt{\frac{P_{1}-F_{F} P_{V}}{G_{F}}}
$$

whee

$F_{L}=0.9$ Appendix D, GLEe VALVE $F_{L}=$ LiQ. Press. Recovers

$P_{1}=$ UPSTREAM PRESSURE, PSIA.

$P_{Z}=$ DOWNSTREAM PRESSURE, PSIA

$P_{v c}=F_{F} P_{v}$

$P_{V}=$ ABS. VAPOR PRESSURE AT INLET

$F_{F}=0.96-0.28\left(\frac{P_{v}}{P_{c}}\right)^{1 / 2}$ Appendix $\dot{P}$.

$P_{C}=\Delta B$ SOLUTE cRITICAL PRESSURE

$G_{f}=$ SPECIFIC GRAvity

SOLUTION

$$
\begin{aligned}
& P_{1}=77.2 \text { PsiA } P_{2}=33 \text { PsiA. } P_{V}=70 \text { PsiA } \quad C_{V}=1.25 \\
& P_{c}=493.13 \text { aSL } \\
& F_{F}=0.96-0.28\left(\frac{70}{493.13}\right)^{1 / 2}=.8545 \\
& P_{V C}=F_{F} P_{V}=.8545(70 . P S / A)=59.815 \mathrm{PSIA} \\
& q_{\text {max }}=(1.0)(.9)(1.25) \sqrt{\frac{77.2-.8545(70)}{. .72 .7^{9 / c c}}}=5.500 \mathrm{gpm}
\end{aligned}
$$

$$
q_{\text {max }}=5.50 \frac{\mathrm{gei}}{\mathrm{mid}} \times .727 \frac{\mathrm{g}}{\mathrm{cm}^{3}} \times \frac{\mathrm{lam}}{60 \mathrm{~s} \cdot} \times \frac{3.785 .4 \mathrm{~cm}^{3}}{252 \mathrm{gal}}=2 / \mathrm{s}
$$

using Anderson Greenwood equations: $V=6.32 \mathrm{gmax} / M$

$$
\text { Vair }=V \sqrt{\text { Mair/MN2 }}
$$

$$
\therefore V_{\text {ain }}=443 \mathrm{SCFm}
$$




\section{References}

1. Introduction to Fluid Mechanics, 3rd Ed., Robert W. Fox, Alan T. McDonald, JohnWiley\&Sons, 1985.

2. "Fike Technical Bulletin TB 8102, Rupture Disk Sizing", Fike Metal Products Corp.

3. “Catalog 1900-Series 90 Safety Relief Valves", Anderson, Greenwood \& Co., 1980.

4. “D $\varnothing \mathrm{CC}$ Pressure Vessel and Vacuum Vessel Safety Note”, D $\varnothing$ Engineering Note \#3740-EN-263, R. Rucinski/R. Luther, Nov., 1990.

5. "Cryostat Filling Limitations for Proposed Ar Dewar Pressure Increase", DØ Engineering Note \#3740-EN-321, J. Wu/K. Dixon, Jul., 1991.

6. "Calorimeter Conduction, Radiation and Fire Loads", D $\varnothing$ Engineering Note \#3740-EN-6, S. J. Wintercorn, May, 1985. 
Prepared By Kelly Dixon Date 20 Ang 91 Div/Sect RD/D $\phi$

Reviewed By

Div/Dept Head Date 10-29-91 Div/Sect AD/Cryoganics Date $10-\angle q-9$, Div/Sect Res Du.

I. Identification and Verification of Compliance

Fill in the Fermilab Engineering Conformance Label information below:

This vessel conforms to Engineering Standard SD-41

vessel Titre North Endcais Calorimeter (NEC) Cryoshut vessel Number RD 9000 5.⿲丿丨.

Vessel Drawing Number $3740.220-M E-222256$

Working Temperature Range $-320^{\circ}{ }^{\circ} 100^{\circ}{ }^{\circ}$

Designer/Manufacturer $\frac{\text { Process thgineering, The }}{\text { Thre } 1990}$

Acceptance Date

Director's signature (or designee) if vessel is for manned area and requires an exception to the provisions of this standard. 


\section{Exhibit A-2}

II. Description of Vessel and Relief System

Laboratory location code

Laboratory property number

Purpose of vessel Essulating jucket for cryostat

List all pertinent drawings (append copies)

Drawing No.:

$3740.220-M E-222256$

$3740.224-M E-273065$

$3740.224-M E-273030$
Location of Original:

DD Files, DAB

DQ Files, DAB

$D \phi$ Files, $D A B$

Can this vessel be pressurized?

If yes, to what pressure.

Is a testing procedure necessary for the safe acceptance testing (proof testing) of this vessel?

If yes, supply the written procedure with this Engineering Note.

Is an operating procedure necessary for the safe operation of this vessel?

If yes, supply the written procedure with this Engineering Note.

List all reliefs and settings. Provide a schematic of the relief system components, and appropriate calculations or test results to prove that overpressurization beyond the maximum allowable internal pressure will not occur.

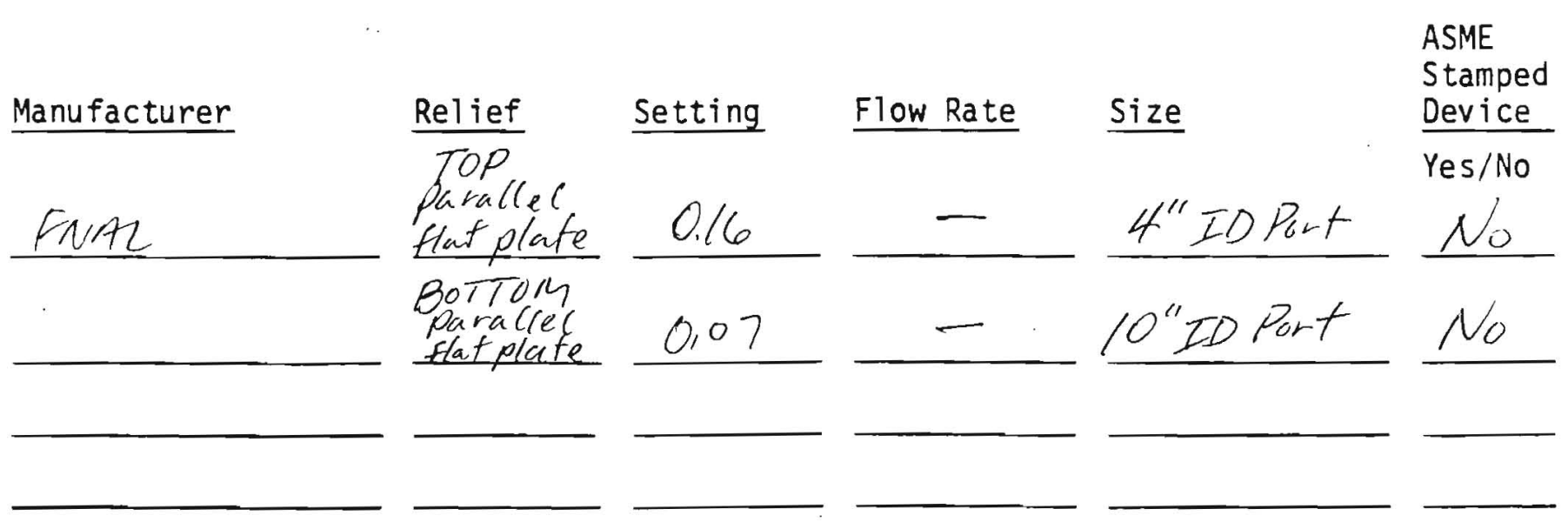

Wel ding Information

Has the vessel been fabricated in a Fermilab shop? Yes No If "Yes", append a copy of the welding shop statement of welder qualification. 


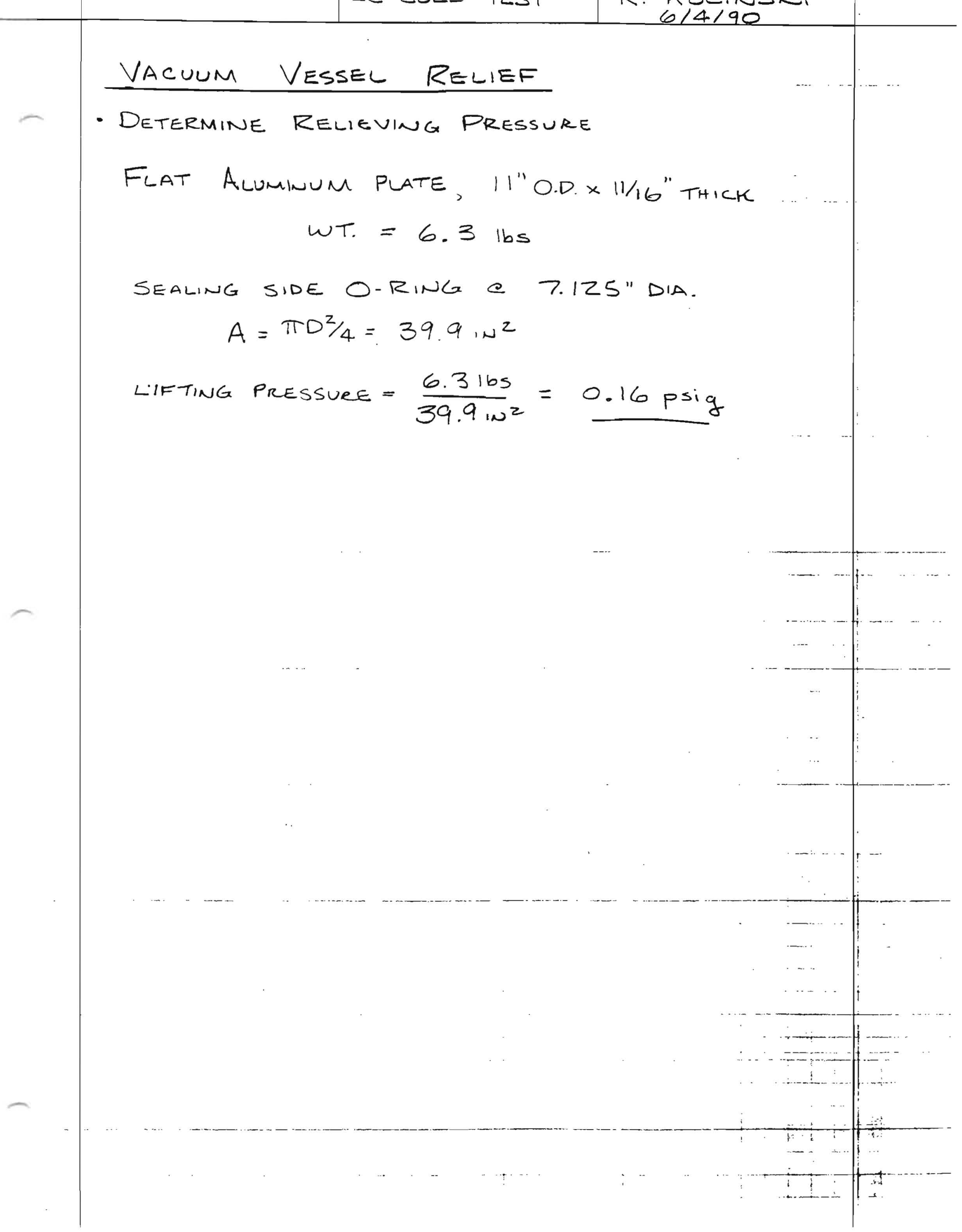


Concussion

Go WITA $15 / 16^{0.0} \times .080$ DI. WIRE SPRING $<2 \frac{1}{32} "$

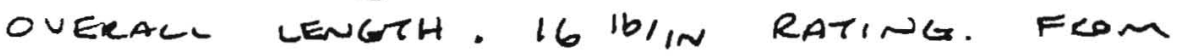
McMatter CArR, $\$ 9658 K 46, \$ 88 /$ each

USE 5 washers, fac STOCK $1218-0720$

And $5 / 8 \times 2^{\prime \prime}$ Stainless STEel ShOULDER GOLTS fROM PRESTIGE PARTS. 1/2-13 THKEAD.

SPRING COMPRESSION WILL BE $078 \times \frac{5}{5}+1 / 32=.42125^{\prime \prime}$ WHICH GIVES IhS FORLE/BOLT 26.96 lbS OVER AL UPWARO FORCE WITH no vacuum.

Relief plate will weigh

$$
21.6 \mathrm{lbs}-\underset{\substack{r \\ \text { Molts }}}{.23}=21.37 \mathrm{lbs}
$$

5.6 lbS Difference up under no vacuUMs

Relief Plate will drop at

$$
\frac{5.6 \mathrm{~b}}{78 . \sin ^{2}}=.07 \text { psid. }
$$


ECN vaccum vessel note calc., 10/10/91

\section{Calculation of Cross-sectional_Area Required for Vacuum Jacket Relief}

References: "Pressure Relief Device Standards", CGA S-1.3-1980

“Standard for Insulated Cargo Tank Specification

for Cryogenic Liquids", CGA-341-1987

Calculate the current area available from the relief devices:

$A_{\text {avail }}^{\text {disc }}=0.785\left(10^{2}+4^{2}\right)=91$ in $^{2}$

From the references, the required area is:

$\mathrm{A}_{\text {req'd }}^{\text {disc }}=0.00024 \mathrm{in}^{2} / 1 \mathrm{~b}_{\mathrm{m}} \mathrm{H}_{2} \mathrm{O}$ capacity

Assume a volume of 3000 gallons of $\mathrm{H}_{2} \mathrm{O}$, as a conservative calculation.

Calculate the total mass of $\mathrm{H}_{2} \mathrm{O}$ :

$3000 \mathrm{gal} \times \frac{0.13368 \mathrm{ft}^{3}}{\mathrm{gal}} \times \frac{62.41 \mathrm{~b}_{\mathrm{m}}}{\mathrm{ft}^{3}}=25,000 \mathrm{lb}_{\mathrm{m}} \mathrm{H}_{2} \mathrm{O}$

Calculate the required area of the reliefs:

$A_{\text {req'd }}^{\text {disc }}=\frac{0.00024 \mathrm{in}^{2}}{1 b_{m} \mathrm{H}_{2} \mathrm{O}} \times 25,0001 b_{m} \mathrm{H}_{2} \mathrm{O}$

$A_{\text {reqd }}^{\text {disc }}=6$ in $^{2}$ 
ECN vaccum vessel note calc., 10/10/91

Since the calculated available area of the reliefs far exceeds the required area, the vacuum vessel is adequately protected from overpressurization. The relief devices installed are parallel plate relief devices (10" and 4"), so the actual area available is not the entire area of the disc, however, the available area calculated is large enough for small errors. 